\title{
Oncogenic value of microRNA-15b-5p in hepatocellular carcinoma and a bioinformatics investigation
}

\author{
WEN-YA PAN ${ }^{1}$, JIANG-HUI ZENG $^{1}$, DONG-YUE WEN ${ }^{2}$, JIE-YU WANG ${ }^{1}$, \\ PENG-PENG WANG ${ }^{3}$, GANG CHEN ${ }^{1}$ and ZHEN-BO FENG ${ }^{1}$
}

\begin{abstract}
Departments of ${ }^{1}$ Pathology, ${ }^{2}$ Ultrasonography and ${ }^{3}$ Nursing, First Affiliated Hospital of Guangxi Medical University, Nanning, Guangxi Zhuang Autonomous Region 530021, P.R. China
\end{abstract}

Received November 9, 2017; Accepted October 12, 2018

DOI: $10.3892 / \mathrm{ol} .2018 .9748$

\begin{abstract}
R-15b-5p has frequently been reported to function as a biomarker in some malignancies; however, the function of miR-15b-5p in hepatocellular carcinoma (HCC) and its molecular mechanism are still not well understood. The present study was designed to confirm the clinical value of miR-15b-5p and further explore its underlying molecular mechanism. A comprehensive investigation of the clinical value of miR-15b-5p in HCC was investigated by data mining The Cancer Genome Atlas (TCGA) and Gene Expression Omnibus (GEO) datasets as well as literature. In addition, intersected target genes of miR-15b-5p were predicted using the miRWalk database and differentially expressed genes of HCC from TCGA. Furthermore, gene ontology (GO) and Kyoto Encyclopedia of Genes and Genomes (KEGG) pathway analyses were carried out. Then, a protein-protein interaction network (PPI) was constructed to reveal the interactions between some hub target genes of miR-15b-5p. The miR-15b-5p expression level in HCC was predominantly overexpressed compared with non-HCC tissues samples (SMD=0.618, 95\% CI: 0.207, 1.029; P<0.0001) based on $991 \mathrm{HCC}$ and 456 adjacent non-HCC tissue samples. The pooled summary receiver operator characteristic (SROC) of miR-15b-5p was $0.81\left(Q^{*}=0.74\right)$, and the pooled sensitivity and specificity of miR-15b-5p in HCC were $72 \%$
\end{abstract}

Correspondence to: Professor Zhen-Bo Feng, Department of Pathology, First Affiliated Hospital of Guangxi Medical University, 6 Shuangyong Road, Nanning, Guangxi Zhuang Autonomous Region 530021, P.R. China

E-mail: fengzhenbo_gxmu@163.com

Abbreviations: miR-15b-5p, microRNA-15b-5p; HCC, hepatocellular carcinoma; GEO, gene expression omnibus; TCGA, The cancer genome atlas; ROC, receiver operating characteristic; AUC, area under curves; GO, gene ontology; KEGG, Kyoto encyclopedia of genes and genomes; PPI, protein-protein interactions

Key words: miR-15b-5p, hepatocellular carcinoma, target genes, TCGA, GEO
(95\% CI: 69-75\%) and 68\% (95\% CI: 65-72\%), respectively. Bioinformatically, 225 overlapping genes were selected as prospective target genes of miR-15b-5p in HCC, and profoundly enriched GO terms and KEGG pathway investigation in silico demonstrated that the target genes were associated with prostate cancer, proximal tubule bicarbonate reclamation, heart trabecula formation, extracellular space, and interleukin-1 receptor activity. Five genes (ACACB, RIPK4, MAP2K1, TLR4 and IGF1) were defined as hub genes from the PPI network. The high expression of miR-15b-5p could play an essential part in hepatocarcinogenesis through diverse regulation approaches.

\section{Introduction}

Hepatocellular carcinoma (HCC), which represents an overwhelming majority of liver cancer, is the sixth most widespread cancer all over the world and the third most common cause of cancer-related deaths (1). As a health threat to people worldwide and, in particular, to developing countries, its overall 5-year survival rate is $5-9 \%$ (2). However, with early diagnosis and curative resection, the 5 -year survival rate can be increased to $69 \%$ (3). Hence, a diagnostic biomarker with high efficacy is urgently needed. In current clinical work, HCC detection often hinges on a-fetoprotein (AFP), which is the most general biomarker for HCC detection. An aberrant high AFP expression level is frequently observed in HCC patients, with a sensitivity of $39-65 \%$ and a specificity of $76-94 \%$ (4). In addition, some researchers have reported that the ability of AFP to identify and differentiate $\mathrm{HCC}$ from non-cancerous hepatopathy is unsatisfying (5-7).

MicroRNAs (miRNAs) are a sequence of short noncoding RNAs that are 20-22 nucleotides in length. They post-transcriptionally regulate the gene level by combining with their target mRNAs and play important administrative roles in a variety of biological processes (8-12). During the past few years, aberrant expression of miRNAs for the early discovery of HCC has been widely verified with inconsistent results. Since then, the significant high expression of miR-15b-5p in HCC patients has been demonstrated by multiple studies $(13,14)$. Liu et al (15) detected miR-15b-5p in HCC with an AUC of 0.485 (98.25\% sensitivity, $15.25 \%$ specificity). In 
another study, Chen et al (16) revealed that the AUC value of miR-15b-5p for HCC detection was 0.654 (68.1\% sensitivity, $79.0 \%$ specificity), 0.871 (87.2\% sensitivity, $74.2 \%$ specificity), and 0.765 (68.1\% sensitivity, $80.0 \%$ specificity), respectively, in subgroups of HCC vs. liver cirrhosis patients, $\mathrm{HCC}$ vs. healthy controls, as well as HCC vs. liver cirrhosis and healthy controls. In fact, the clinical effects of miR-15b-5p on HCC have been reported by only three research groups: i) Hung et al (14) first analyzed the role of miR-15b-5p in the early diagnosis of HCC. They found that when dysplastic nodules (DN) progressed to HCC $(n=10)$, miR-15b-5p levels significantly increased and the serum level of miR-15b-5p in 120 patients with early HCC was also upregulated as compared to that of 30 patients with chronic hepatitis $\mathrm{B}$ using reverse transcription-quantitative polymerase chain reaction (RT-qPCR). ii) Liu et al (15) detected the serum levels of 29 hepatitis B carriers, 57 patients with HCC and 30 healthy controls also using RT-qPCR. They revealed that the expression of miR-15b-5p was significantly higher in all HCC samples. iii) Chen et al (16) detected the expression level of miR-15b-5p in 37 patients with HCC, 29 patients with cirrhosis, and 31 healthy controls by RT-qPCR, and the results revealed that the plasma levels of miR-15b-5p in HCC patients were higher than in the other 2 groups $(\mathrm{P}<0.05)$. However, these studies were inconsistent their miR-15b-5p multiple ability indexes. To improve this issue, a meta-analysis of miR-15b-5p in patients with HCC grounded in data gathered from the Gene Expression Omnibus (GEO; https://www.ncbi.nlm.nih.gov/geo/) and The Cancer Genome Atlas (TCGA; https://cancergenome.nih.gov/) were utilized to evaluate the clinical effectiveness of miR-15b-5p. Bioinformatics analyses were also utilized to investigate the mechanism of miR-15b-5p in HCC. The framework of this study is displayed in Fig. 1.

\section{Materials and methods}

Excavation of TCGA and GEO. In TCGA, HCC-related resources with entries named liver hepatocellular carcinoma (LIHC) were downloaded, correlated miRNA-Seq profiles were provided and miR-15b-5p levels were extracted. To normalize the expression level of miR-15b-5p in different trials, data were $\log _{2}$-scaled afterwards. Expression data sets of miR-15b-5p were attained from the GEO database, and the expression levels of miR-15b-5p in other types of hepatic tissues were also gathered as the control group as well as miR-15b-5p in HCC. Aimed at more precisely assessing the potential value of miR-15b-5p, the inclusion criteria extended to various non-cancerous samples. Microarrays concerning cell lines and other species were excluded because they did not conform to our study. Based on the expression levels of miR-15b-5p in GEO and TCGA, GraphPad Prism 6.0 (GraphPad Software, Inc., La Jolla, CA, USA) was used to generate the scatter plots and ROC. Additionally, HCC patients obtained from TCGA were utilized to analyze the corresponding clinical information.

Literature search. Relevant literature was read and gathered from PubMed (https://www.ncbi.nlm.nih.gov/pubmed/), Web of Science (https://clarivate.com/products/web-of-science/),
Ovid(http://www.ovid.com/site/index.jsp),EBSCO(https://www. ebsco.com/products/research-databases), Embase (https://www.elsevier.com/solutions/embase-biomedical-research), Cochrane Library (https://www.cochranelibrary.com/), Chinese CNKI (http://www.cnki.net/), China Biology Medicine disc, the Chinese Chong Qing VIP (http://en.cqvip. com/), and the Chinese Wan Fang (http://eng.med.wanfangdata. com.cn/). No language limitations were imposed. The following combination of terms with two sets of keywords were screened by combining the underlying searching strategies: (miR-15b OR miRNA-15b OR micrORNA-15b OR miR15b OR miRNA15b OR microRNA15b OR 'miR 15b' OR 'miRNA 15b' OR 'microRNA 15b' OR miR-15b-5p OR miRNA-15b-5p OR micrORNA-15b-5p OR micrORNA-15b OR miR-15b-5p) AND (malignan* OR cancer OR tumor OR tumour OR neoplas* OR carcinoma) AND (hepatocellular cancer OR hepatocellular tumor OR hepatocellular carcinoma OR hepatocellular neoplasm OR liver cancer OR liver tumor OR liver carcinoma OR liver neoplasm OR HCC). Human studies were limited to our literature searches. To confirm the qualified studies, the relevant studies and other references of the review papers were included to avoid missing related studies. Sufficient HCC data and control groups were required to obtain true positives (TPs), false positives (FPs), false negatives (FNs), and true negatives (TNs).

Potential target gene collection and bioinformatics analyses. To further investigate the regulatory mechanism of miR-15b-5p in HCC, MiRWalk2.0 (http://zmf.umm.uni-heidelberg. de/apps/zmf/mirwalk2/), which combines 12 online prediction programs, was used to provide comprehensive potential targets for miR-15b-5p. Genes identified by $>4$ prediction software programs for miR-15b-5p were selected to obtain more reliable targets. The selected predicted target genes were further intersected with TCGA differentially expressed genes. The overlapping genes were considered to be potential target genes of miR-15b-5p. We combined the two parts of the target genes of miR-15b-5p for further gene functional enrichment analyses. In addition, the genes were input to the STRING version 10.0 online tool (http://string-db.org/) to construct the protein-protein interaction (PPI) network.

DAVID 6.8 (https://david.ncifcrf.gov/) was applied for gene ontology (GO; http://geneontology.org/) and Kyoto Encyclopedia of Genes and Genomes (KEGG; https://www. genome.jp/kegg/) pathway analysis. GO was composed of three sections: Molecular function (MF), cellular component (CC) and biological process (BP). The top ten terms of each GO category and marked KEGG pathways were visualized as GO maps and KEGG maps $(17,18)$. Protein expression of hub genes was validated by The Human Protein Atlas (HPA; www. proteinatlas.org), an immunohistochemistry (IHC) database. The IHC images are publicly available.

Diagnostic test and statistical analysis. Diagnostic tests were conducted to assess the efficacy of miR-15b-5p in HCC. To thoroughly determine its clinical potential, analyses were carried out between HCC patients and controls, including healthy controls, non-cancerous controls, adjacent non-neoplastic hepatic tissues, $\mathrm{HBV}^{+} / \mathrm{HCV}^{+}$controls and liver cirrhosis controls. The efficiency of miR-15b-5p 


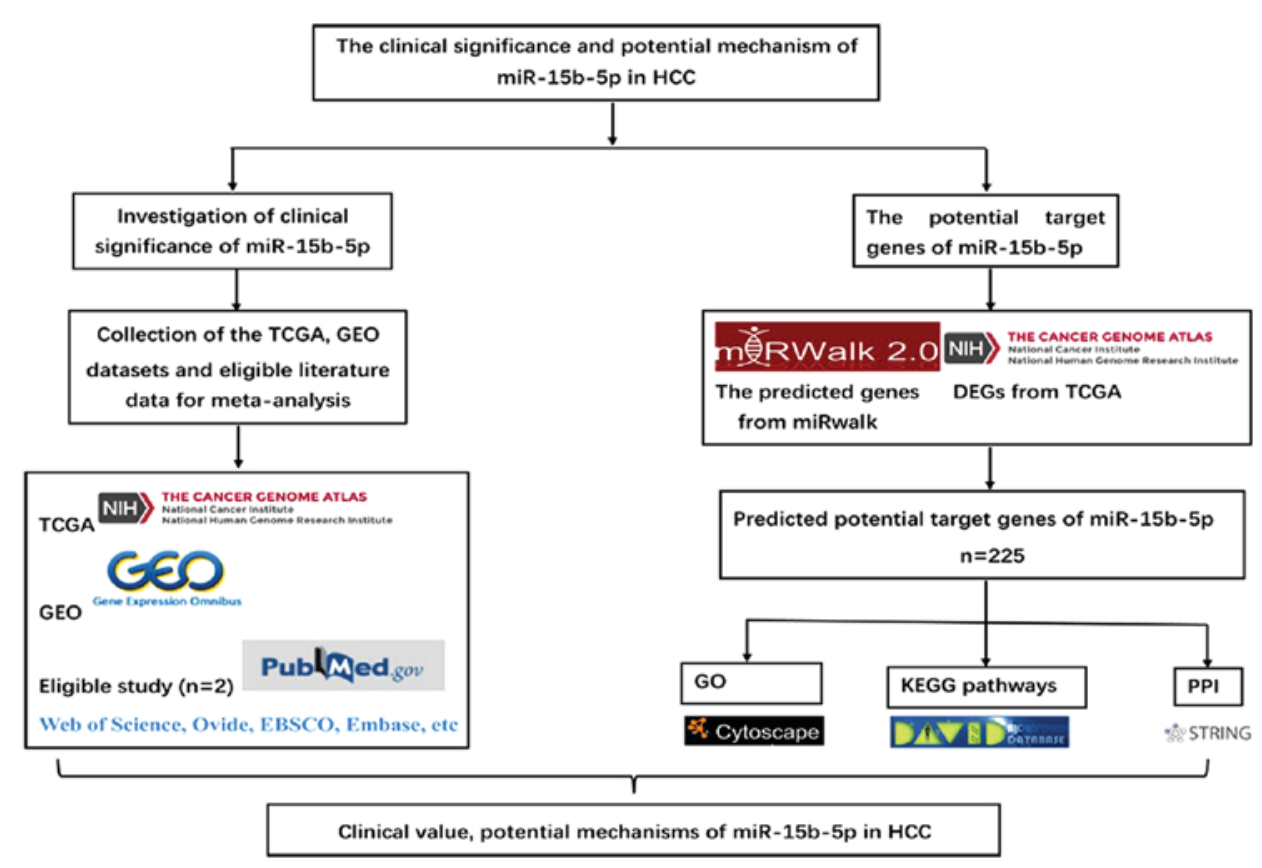

Figure 1. Framework of the present study.

in the serum/plasma and tissues was also examined. Stata 12.0 (https://www.stata.com/stata12/) was used to detect publication bias, and the standard mean difference (SMD) was used to calculate the outcome from GEO and TCGA. The remaining analyses were accomplished by Meta-DiSc 1.4. $\mathrm{P}<0.05$ was recognized as statistically significant. The summary receiver operator characteristic (SROC) curve was plotted according to the included studies. A meta-analysis was carried out using a random effects model, including SEN, SEP, diagnostic odds ratio (DOR), positive likelihood ratio (PLR), and negative likelihood ratio (NLR). In addition, the $\mathrm{I} 2$ index and $\chi^{2}$ test were used to evaluate the heterogeneity in the study. If the $\mathrm{I}^{2}$ value was over $50 \%$ or P-values of the $\chi^{2}$ test were $<0.05$, heterogeneity would be shown. Finally, Deek's funnel plot was displayed to assess the publication bias.

\section{Results}

Qualified studies and dataset. A total of 3,310 relevant articles were acquired from the aforementioned online databases by a primary search. After removing the duplicated articles as well as screening titles, abstracts and full texts, 2 studies were eventually included, and both were published in English $(15,19)$. The chosen studies provided data from 114 HCC patients and 119 people as controls.

According to our criteria, 11 microarray datasets from GEO were evaluated as eligible, consisting of 512 HCC tissue samples and 287 control samples. Sequencing data in TCGA were based upon 425 samples, with 375 diagnosed HCC samples and 50 control samples, as shown in Table I (20-27).

Overall assessment of the diagnostic value and diagnostic meta-analysis. For a more comprehensive understanding of the efficiency of miR-15b-5p in HCC, the eligible 11 GSE chips that we searched were included in our meta-analysis.
The expression levels that correlated with miR-15b-5p in GEO and TCGA were also displayed in Fig. 2. TCGA and 11 GEO profiles with an AUC were presented in Fig. 3. To further explore the clinicopathological features of miR-15b-5p in TCGA, all of the clinicopathological features mentioned in the chips were collected to investigate their correlation with the miR-15b-5p expression level, the results of which are provided in Table II. No noteworthy relationships were observed between miR-15b-5p expression and the clinicopathological characteristics. Compared with the non-neoplastic controls, the miR-15b-5p levels in HCC revealed that the pooled AUC, SEN, SPE, PLR, NLR and DOR were $0.81\left(\mathrm{Q}^{*}=0.74\right), 0.72$ (95\% CI: 0.69-0.75), 0.68 (95\% CI: 0.65-0.72), 3.18 (95\% CI: 1.83-5.51), 0.43 (95\% CI: 0.35-0.54), and 8.98 (95\% CI: 4.30-18.76) (Fig. 4), Furthermore, subgroup analyses with both SMD and sROC methods were performed. The subgroups included sample sources (tissues and serum/plasma) and control types (healthy controls, adjacent non-cancerous hepatic tissues, $\mathrm{HBV}^{+}$or $\mathrm{HCV}^{+}$tissues, liver cirrhosis tissues, and those combining $\mathrm{HBV}^{+} / \mathrm{HCV}^{+}$and cirrhosis). The results were presented from Fig. 5 to Fig. 12. The control types in healthy people and $\mathrm{HBV}^{+}$or $\mathrm{HCV}^{+}$patients had a favorable diagnostic accuracy with AUC-SROC over 0.9, respectively, when in tissues, serum/plasma, adjacent non-cancerous hepatic tissues, liver cirrhosis and combined $\mathrm{HBV}^{+} / \mathrm{HCV}^{+}$and cirrhosis were used as the control. The AUC in the ROC curve was over 0.7.

With the random-effects model, forest-plots were generated to represent significant differences in expression between HCC and non-neoplastic control tissues. The pooled SMD $(0.62,95 \%$ CI: $0.21,1.03)$ is presented in Fig. 12A. The results of pooled SMD between HCC and tissues, healthy subjects, adjacent non-cancerous hepatic tissues, $\mathrm{HBV}^{+}$or $\mathrm{HCV}^{+}$patients, liver cirrhosis, and $\mathrm{HBV}^{+} / \mathrm{HCV}^{+}$combined with cirrhosis were displayed in Fig. 12. The expression level of miR-15b-5p in HCC samples was markedly overexpressed 


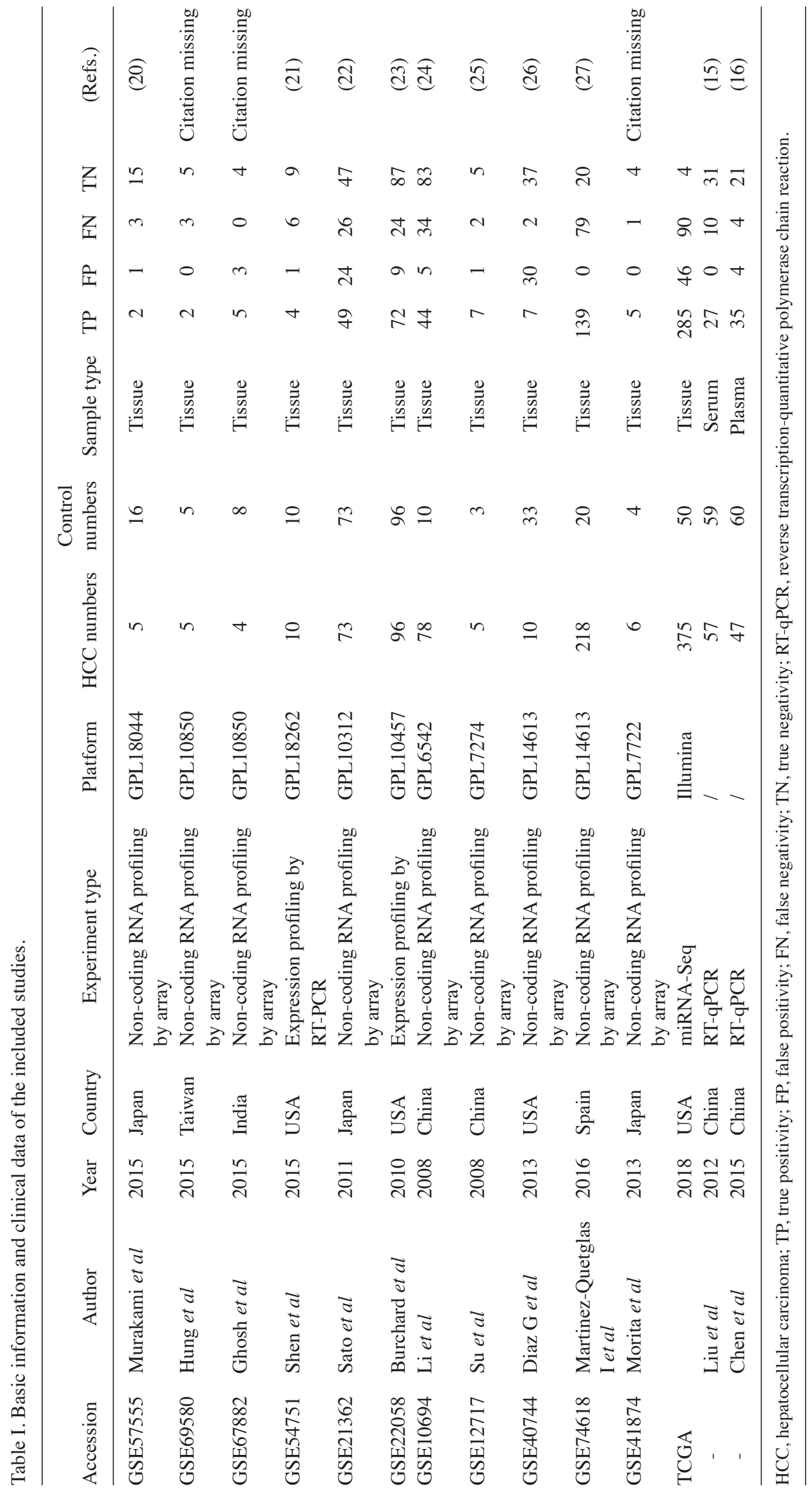


Table II. Relationship between the levels of miR-15b-5p and clinicopathological variables in HCC from the TCGA database.

\begin{tabular}{|c|c|c|c|c|}
\hline Parameters & $\mathrm{N}$ & Mean value & T-value & P-value \\
\hline \multicolumn{5}{|l|}{ Group } \\
\hline $\mathrm{HCC}$ & 375 & $10.128 \pm 0.796$ & \multirow[t]{2}{*}{-4.331} & \multirow[t]{2}{*}{$<0.001$} \\
\hline Normal & 50 & $9.823 \pm 0.402$ & & \\
\hline \multicolumn{5}{|l|}{ Sex } \\
\hline Male & 254 & $10.072 \pm 0.973$ & \multirow[t]{2}{*}{1.069} & \multirow[t]{2}{*}{0.009} \\
\hline Female & 122 & $9.886 \pm 1.802$ & & \\
\hline \multicolumn{5}{|l|}{ Tumor status } \\
\hline With tumor & 152 & $10.207 \pm 0.818$ & \multirow[t]{2}{*}{-1.673} & \multirow[t]{2}{*}{0.527} \\
\hline Tumor-free & 201 & $10.066 \pm 0.764$ & & \\
\hline \multicolumn{5}{|l|}{ Age (years) } \\
\hline$<60$ & 170 & $10.100 \pm 0.778$ & \multirow[t]{2}{*}{-0.424} & \multirow[t]{2}{*}{0.711} \\
\hline$\geq 60$ & 201 & $10.134 \pm 0.799$ & & \\
\hline \multicolumn{5}{|l|}{ Race } \\
\hline Caucasian & 182 & $10.074 \pm 0.819$ & \multirow[t]{2}{*}{-1.199} & \multirow[t]{2}{*}{0.562} \\
\hline Asian & 161 & $10.176 \pm 0.742$ & & \\
\hline \multicolumn{5}{|l|}{ TNM Stage } \\
\hline I-II & 258 & $10.119 \pm 0.767$ & \multirow[t]{2}{*}{0.346} & \multirow[t]{2}{*}{0.203} \\
\hline III-IV & 90 & $10.085 \pm 0.859$ & & \\
\hline \multicolumn{5}{|c|}{ Pathological Stage } \\
\hline G1-2 & 231 & $10.054 \pm 0.754$ & \multirow[t]{2}{*}{-2.037} & \multirow[t]{2}{*}{0.922} \\
\hline G3-4 & 137 & $10.226 \pm 0.832$ & & \\
\hline \multicolumn{5}{|l|}{ T stage } \\
\hline $\mathrm{T} 1-2$ & 276 & $10.111 \pm 0.775$ & \multirow[t]{2}{*}{-0.471} & \multirow[t]{2}{*}{0.477} \\
\hline $\mathrm{T} 3-4$ & 93 & $10.156 \pm 0.833$ & & \\
\hline
\end{tabular}

HCC, hepatocellular carcinoma; TCGA, The Cancer Genome Atlas; TNM, tumor-node-metastasis.

Table III. KEGG functional annotation for most significantly related targets of miR-15b-5p.

\begin{tabular}{llrr}
\hline Category & \multicolumn{1}{c}{ Term } & Count & P-value \\
\hline KEGG_PATHWAY & hsa05215: Prostate cancer & 7 & $1.56 \times 10^{-3}$ \\
& hsa04964: Proximal tubule bicarbonate reclamation & $4.10 \times 10^{-3}$ \\
& hsa04610: Complement and coagulation cascades & 5 & $1.68 \times 10^{-2}$ \\
& hsa01100: Metabolic pathways & 27 & $2.18 \times 10^{-2}$ \\
& hsa00280: Valine, leucine and isoleucine degradation & 8 & $3.06 \times 10^{-2}$ \\
& hsa04015: Rap1 signaling pathway & $3.26 \times 10^{-2}$ \\
& hsa00220: Arginine biosynthesis & 6 & $3.28 \times 10^{-2}$ \\
& hsa04068: FoxO signaling pathway & 6 &
\end{tabular}

KEGG, Kyoto Encyclopedia of Genes and Genomes.

than in non-HCC tissues samples. Moreover, the Deek's funnel plot asymmetry test was carried out with STATA 12.0, and no publication bias was detected apart from healthy people and $\mathrm{HBV}^{+}$or $\mathrm{HCV}^{+}$patients. $(\mathrm{P}<0.05)$ (Fig. 13).

Potential target genes and bioinformatics annotation. Nine thousand seven-hundred-eighty target genes appearing $\geq 4$ times in 12 prediction methods were regarded as probable target genes of miR-15b-5p from miRWalk. Furthermore, downregulated expressed genes assembled from TCGA were integrated to generate the intersection of target genes, which had more potential to be the real targets of miR-15b-5p in HCC. After 9,780 potential target genes and 1,123 TCGA differentially expressed genes were analyzed for intersection, 
Table IV. GO functional annotation of the target genes of miR-15b-5p.

\begin{tabular}{|c|c|c|c|c|}
\hline GO ID & Category & GO term & P-value & Count \\
\hline GO:0060347 & GO_Biological process & Heart trabecula formation & $5.26 \times 10^{-4}$ & 4 \\
\hline GO:0001657 & GO_Biological process & Ureteric bud development & $9.93 \times 10^{-4}$ & 5 \\
\hline GO:0006520 & GO_Biological process & Cellular amino acid metabolic process & $1.21 \times 10^{-3}$ & 5 \\
\hline GO:0032354 & GO_Biological process & Response to follicle-stimulating hormone & $1.34 \times 10^{-3}$ & 3 \\
\hline GO:0000187 & GO_Biological process & Activation of MAPK activity & $1.64 \times 10^{-3}$ & 7 \\
\hline GO:0032754 & GO_Biological process & Positive regulation of interleukin-5 production & $1.99 \times 10^{-3}$ & 3 \\
\hline GO:0006814 & GO_Biological process & Sodium ion transport & $2.63 \times 10^{-3}$ & 6 \\
\hline GO:0010951 & GO_Biological process & Negative regulation of endopeptidase activity & $3.04 \times 10^{-3}$ & 7 \\
\hline GO:0008652 & GO_Biological process & Cellular amino acid biosynthetic process & $3.39 \times 10^{-3}$ & 4 \\
\hline GO:0019221 & GO_Biological process & Cytokine-mediated signaling pathway & $4.49 \times 10^{-3}$ & 7 \\
\hline GO:0005615 & GO_Cellular component & Extracellular space & $4.20 \times 10^{-3}$ & 28 \\
\hline GO:0005759 & GO_Cellular component & Mitochondrial matrix & $5.27 \times 10^{-3}$ & 11 \\
\hline GO:0005886 & GO_Cellular component & Plasma membrane & $6.97 \times 10^{-3}$ & 65 \\
\hline GO:0005887 & GO_Cellular component & Integral component of plasma membrane & $7.97 \times 10^{-3}$ & 28 \\
\hline GO:0005578 & GO_Cellular component & Proteinaceous extracellular matrix & $1.37 \times 10^{-2}$ & 9 \\
\hline GO:0005576 & GO_Cellular component & Extracellular region & $2.18 \times 10^{-2}$ & 29 \\
\hline GO:0009986 & GO_Cellular component & Cell surface & $2.59 \times 10^{-2}$ & 13 \\
\hline GO:0045211 & GO_Cellular component & Postsynaptic membrane & $3.79 \times 10^{-2}$ & 7 \\
\hline GO:0005739 & GO_Cellular component & Mitochondrion & $3.85 \times 10^{-2}$ & 24 \\
\hline GO:0009897 & GO_Cellular component & External side of plasma membrane & $3.93 \times 10^{-2}$ & 7 \\
\hline GO:0004908 & GO_Molecular function & Interleukin-1 receptor activity & $2.79 \times 10^{-3}$ & 3 \\
\hline GO:0008201 & GO_Molecular function & Heparin binding & $2.91 \times 10^{-3}$ & 8 \\
\hline GO:0004867 & GO_Molecular function & Serine-type endopeptidase inhibitor activity & $5.81 \times 10^{-3}$ & 6 \\
\hline GO:0042803 & GO_Molecular function & Protein homodimerization activity & $5.89 \times 10^{-3}$ & 18 \\
\hline GO:0001078 & GO_Molecular function & $\begin{array}{l}\text { Transcriptional repressor activity, RNA polymerase II core } \\
\text { promoter proximal region sequence-specific binding }\end{array}$ & $1.01 \times 10^{-2}$ & 6 \\
\hline GO:0005088 & GO_Molecular function & Ras guanyl-nucleotide exchange factor activity & $1.17 \times 10^{-2}$ & 6 \\
\hline GO:0004860 & GO_Molecular function & Protein kinase inhibitor activity & $2.34 \times 10^{-2}$ & 4 \\
\hline GO:0002114 & GO_Molecular function & Interleukin-33 receptor activity & $2.34 \times 10^{-2}$ & 2 \\
\hline GO:0004854 & GO_Molecular function & Xanthine dehydrogenase activity & $2.34 \times 10^{-2}$ & 2 \\
\hline GO:0051537 & GO_Molecular function & 2 iron, 2 sulfur cluster binding & $3.47 \times 10^{-2}$ & 3 \\
\hline
\end{tabular}

GO, gene ontology.

Table V. GO functional annotation of the hub genes of miR-15b-5p in HCC.

\begin{tabular}{|c|c|c|c|c|}
\hline GO ID & Category & Term & P-value & Count \\
\hline GO:0000187 & GOTERM_BP & Activation of MAPK activity & $2.39 \times 10^{-4}$ & 3 \\
\hline GO:0070371 & GOTERM_BP & ERK1 and ERK2 cascade & $5.71 \times 10^{-3}$ & 2 \\
\hline GO:0006928 & GOTERM_BP & Movement of cell or subcellular component & $2.03 \times 10^{-2}$ & 2 \\
\hline GO:0051092 & GOTERM_BP & Positive regulation of $\mathrm{NF}-\kappa \mathrm{B}$ transcription factor activity & $3.13 \times 10^{-2}$ & 2 \\
\hline GO:0010629 & GOTERM_BP & Negative regulation of gene expression & $3.22 \times 10^{-2}$ & 2 \\
\hline GO:0070374 & GOTERM_BP & Positive regulation of ERK1 and ERK2 cascade & $4.10 \times 10^{-2}$ & 2 \\
\hline GO:0010628 & GOTERM_BP & Positive regulation of gene expression & $6.10 \times 10^{-2}$ & 2 \\
\hline GO:0005524 & GOTERM_MF & ATP binding & $4.17 \times 10^{-2}$ & 3 \\
\hline GO:0005515 & GOTERM_MF & Protein binding & $7.33 \times 10^{-2}$ & 5 \\
\hline GO:0004672 & GOTERM_MF & Protein kinase activity & $8.24 \times 10^{-2}$ & 2 \\
\hline GO:0004674 & GOTERM_MF & Protein serine/threonine kinase activity & $8.62 \times 10^{-2}$ & 2 \\
\hline
\end{tabular}

GO, gene ontology; HCC, hepatocellular carcinoma; BP, biological process; MF, molecular function; CC, cellular component. 
Table VI. KEGG pathway analysis of the hub genes of miR-15b-5p in HCC.

\begin{tabular}{lllll}
\hline Category & Term & Count & P-value & Genes \\
\hline KEGG_PATHWAY & hsa04066: HIF-1 signaling pathway & 3 & $5.92 \times 10^{-4}$ & MAP2K1, IGF1, TLR4 \\
KEGG_PATHWAY & hsa05205: Proteoglycans in cancer & 3 & $2.45 \times 10^{-3}$ & MAP2K1, IGF1, TLR4 \\
KEGG_PATHWAY & hsa04151: PI3K-Akt signaling pathway & 3 & $7.21 \times 10^{-3}$ & MAP2K1, IGF1, TLR4 \\
KEGG_PATHWAY & hsa04730: Long-term depression & 2 & $2.58 \times 10^{-2}$ & MAP2K1, IGF1 \\
KEGG_PATHWAY & hsa05214: Glioma & 2 & $2.80 \times 10^{-2}$ & MAP2K1, IGF1 \\
KEGG_PATHWAY & hsa05218: Melanoma & 2 & $3.05 \times 10^{-2}$ & MAP2K1, IGF1 \\
KEGG_PATHWAY & hsa04914: Progesterone-mediated oocyte maturation & 2 & $3.73 \times 10^{-2}$ & MAP2K1, IGF1 \\
KEGG_PATHWAY & hsa05215: Prostate cancer & 2 & $3.77 \times 10^{-2}$ & MAP2K1, IGF1 \\
KEGG_PATHWAY & hsa04620: Toll-like receptor signaling pathway & 2 & $4.53 \times 10^{-2}$ & MAP2K1, TLR4 \\
KEGG_PATHWAY & hsa04114: Oocyte meiosis & 2 & $4.66 \times 10^{-2}$ & MAP2K1, IGF1
\end{tabular}

KEGG, Kyoto Encyclopedia of Genes and Genomes; HCC, hepatocellular carcinoma.
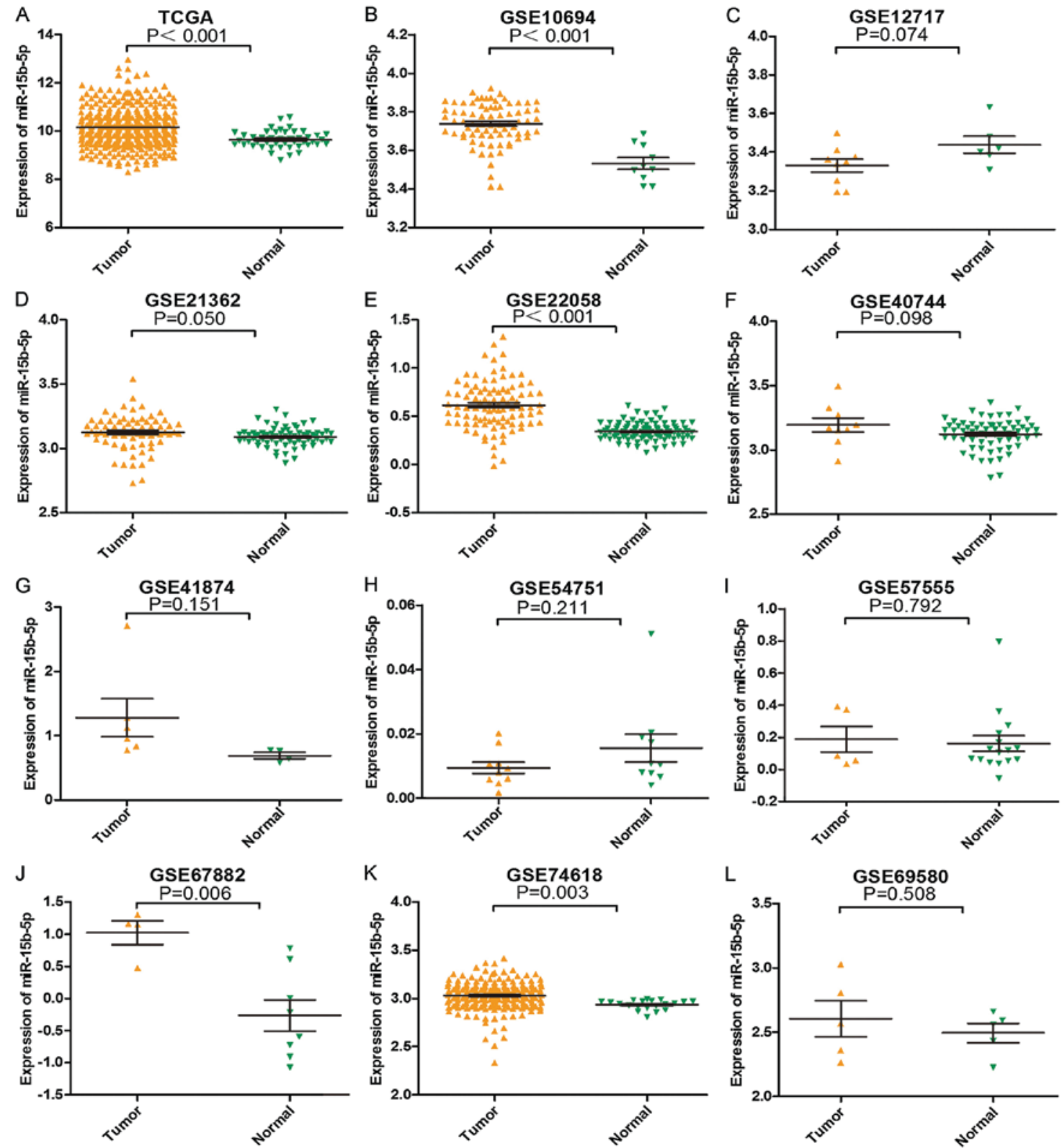

Figure 2. Expression level of each study from TCGA and GEO. (A) TCGA, (B) GSE10694, (C) GSE12717, (D) GSE21362, (E) GSE22058, (F) GSE40744, (G) GSE41874, (H) GSE54751, (I) GSE57555, (J) GSE67882, (K) GSE74618 and (L) GSE69580. 

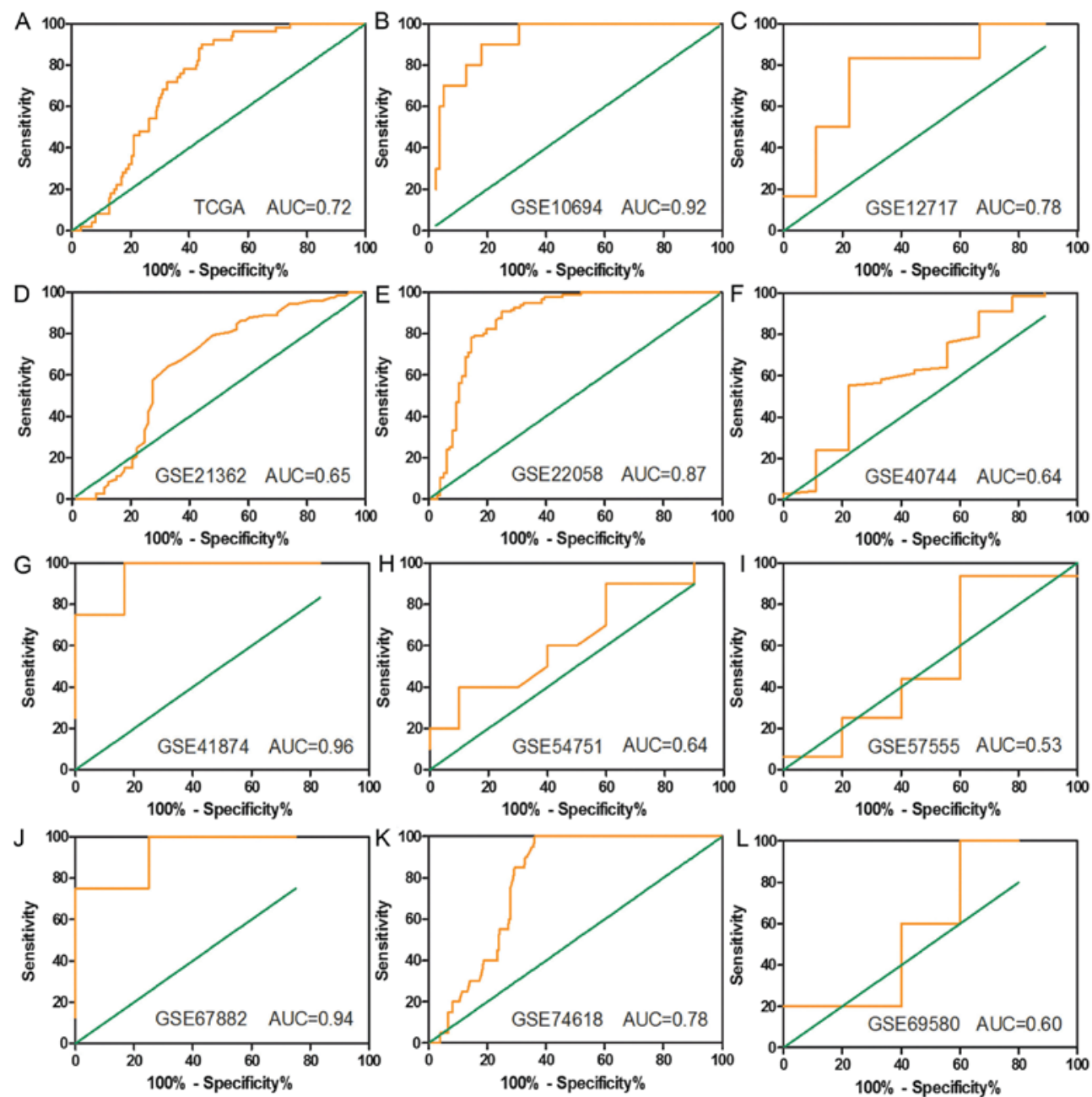

Figure 3. Receiver operating characteristic (ROC) curves based on the TCGA and GEO datasets. (A) TCGA, (B) GSE10694, (C) GSE12717, (D) GSE21362, (E) GSE22058, (F) GSE40744, (G) GSE41874, (H) GSE54751, (I) GSE57555, (J) GSE67882, (K) GSE74618 and (L) GSE69580.
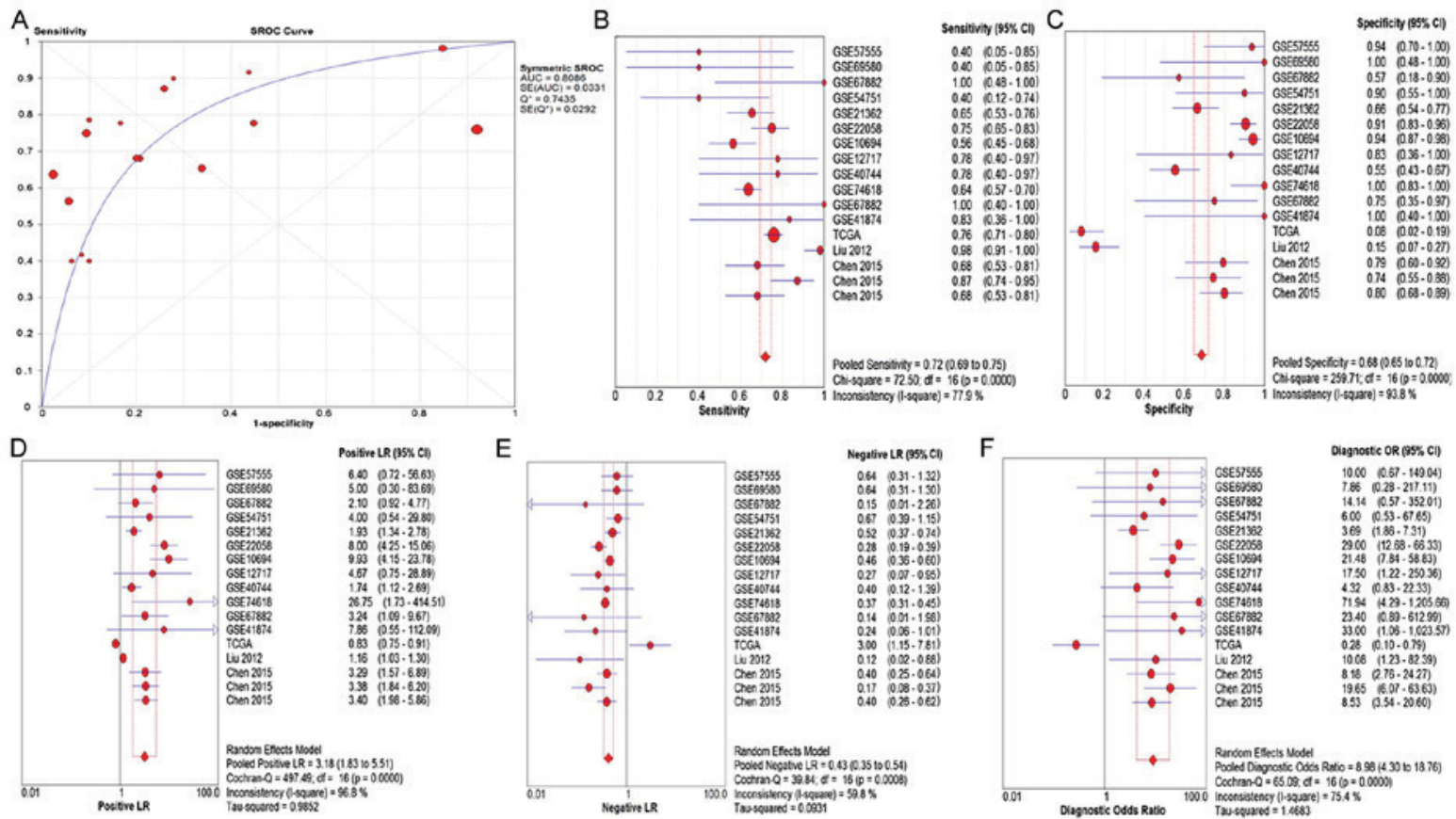

Figure 4. The pooled (A) SROC curve, (B) SEN, (C) SPE, (D) PLR, (E) NLR and (F) DOR analysis of the qualified studies of miR-15b-5p in the HCC group compared with the control group. SROC, summarized receiver operating characteristic; SEN, sensitivity; SPE, specificity; PLR, positive likelihood ratio; NLR; negative likelihood ratio; DOR, diagnostic odds ratio; HCC, hepatocellular carcinoma. 


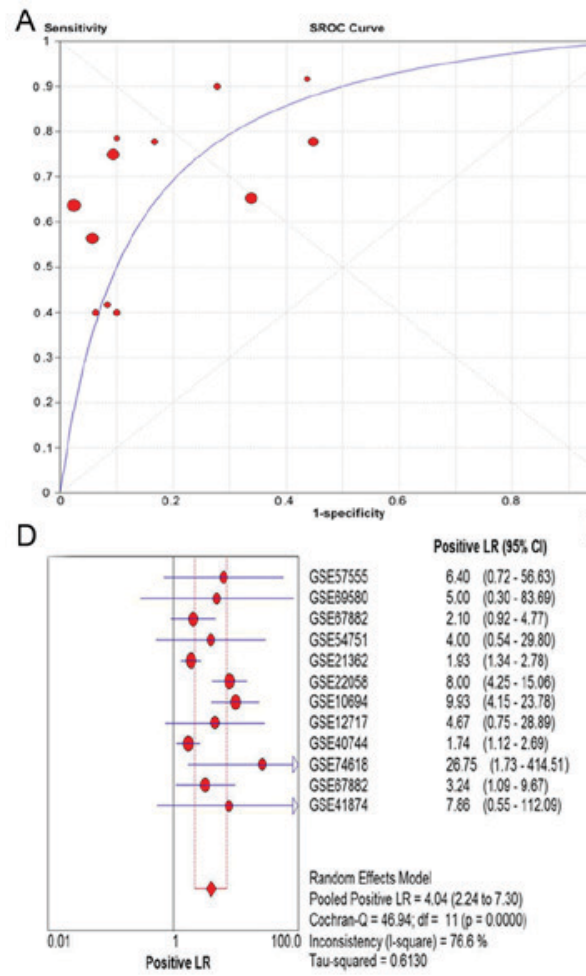

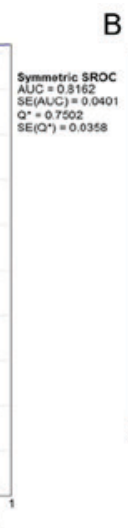

$\mathrm{E}$

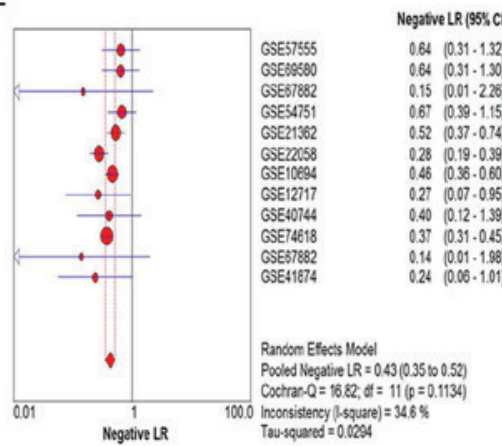

B

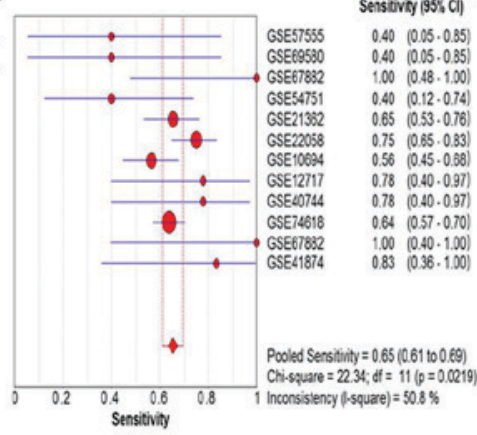

C

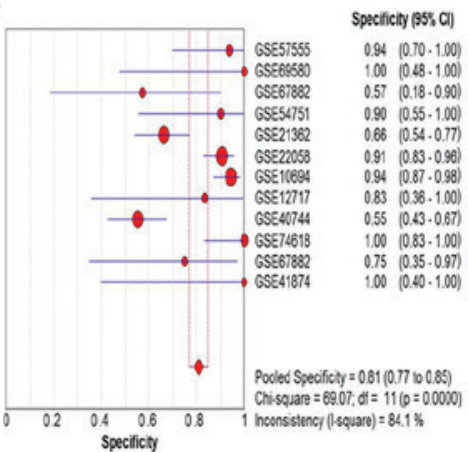

$\mathrm{F}$

Figure 5. The pooled (A) SROC curve, (B) SEN, (C) SPE, (D) PLR, (E) NLR and (F) DOR of miR-15b-5p in tissues. SROC, summarized receiver operating characteristic; SEN, sensitivity; SPE, specificity; PLR, positive likelihood ratio; NLR, negative likelihood ratio; DOR, diagnostic odds ratio.

A

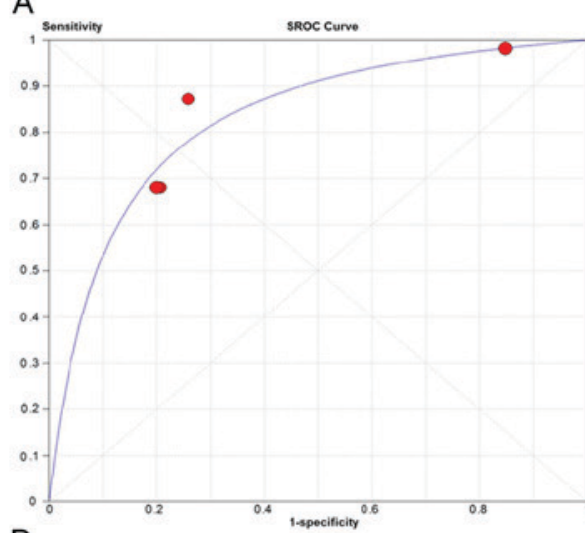

D

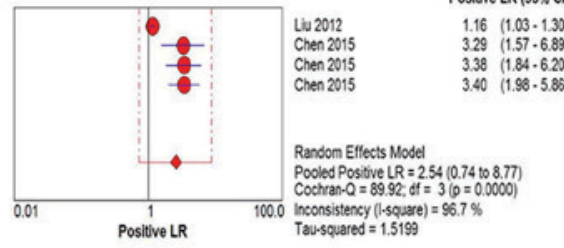

B

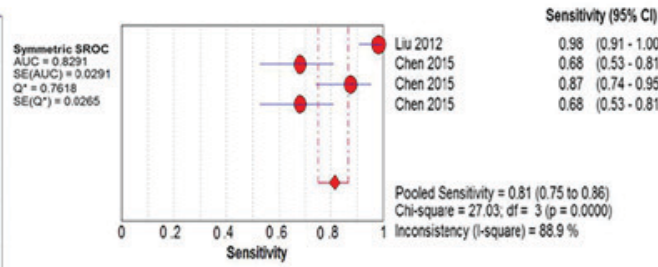

C

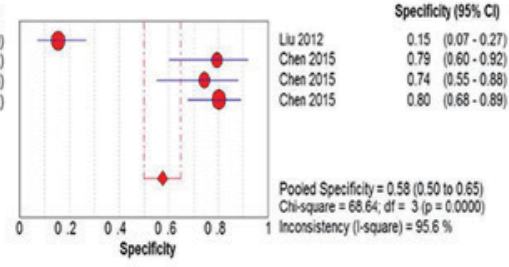

Dagnostic OR (355 Cl)

$1000 \cdot(0.67 \cdot 149.04)$ $7.86(0.26-217.11)$ $1414 \quad 0.57 \cdot 352.01$ $6.00(0.53 \cdot 67.65)$ 2900 (1268.66.33) 21.48 (7.84-58.83) $17.50(1.22 \cdot 250.38)$ $7194 \quad(429-120566)$ $2340(0.89-612.99)$

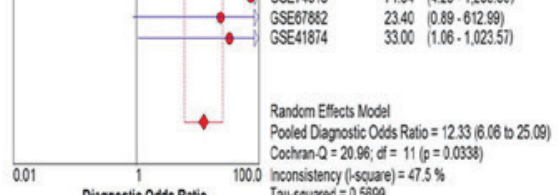

Diagnossic Odats Ratio 100.0 heonsistency $(1-$ scuare $)=47.5 \%$

25.09)




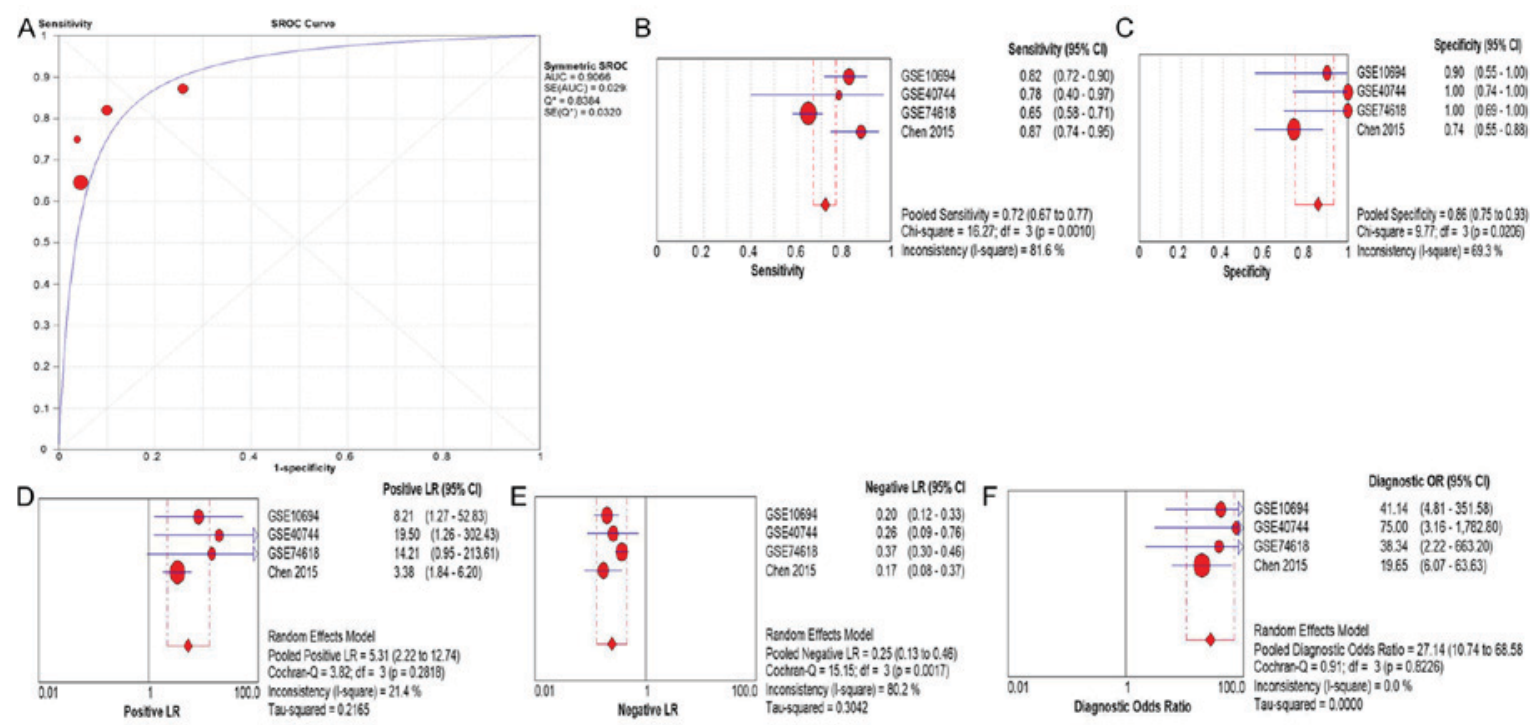

Figure 7. The pooled (A) SROC curve, (B) SEN, (C) SPE, (D) PLR, (E) NLR and (F) DOR of miR-15b-5p in healthy people. SROC, summarized receiver operating characteristic; SEN, sensitivity; SPE, specificity; PLR, positive likelihood ratio; NLR, negative likelihood ratio; DOR, diagnostic odds ratio.

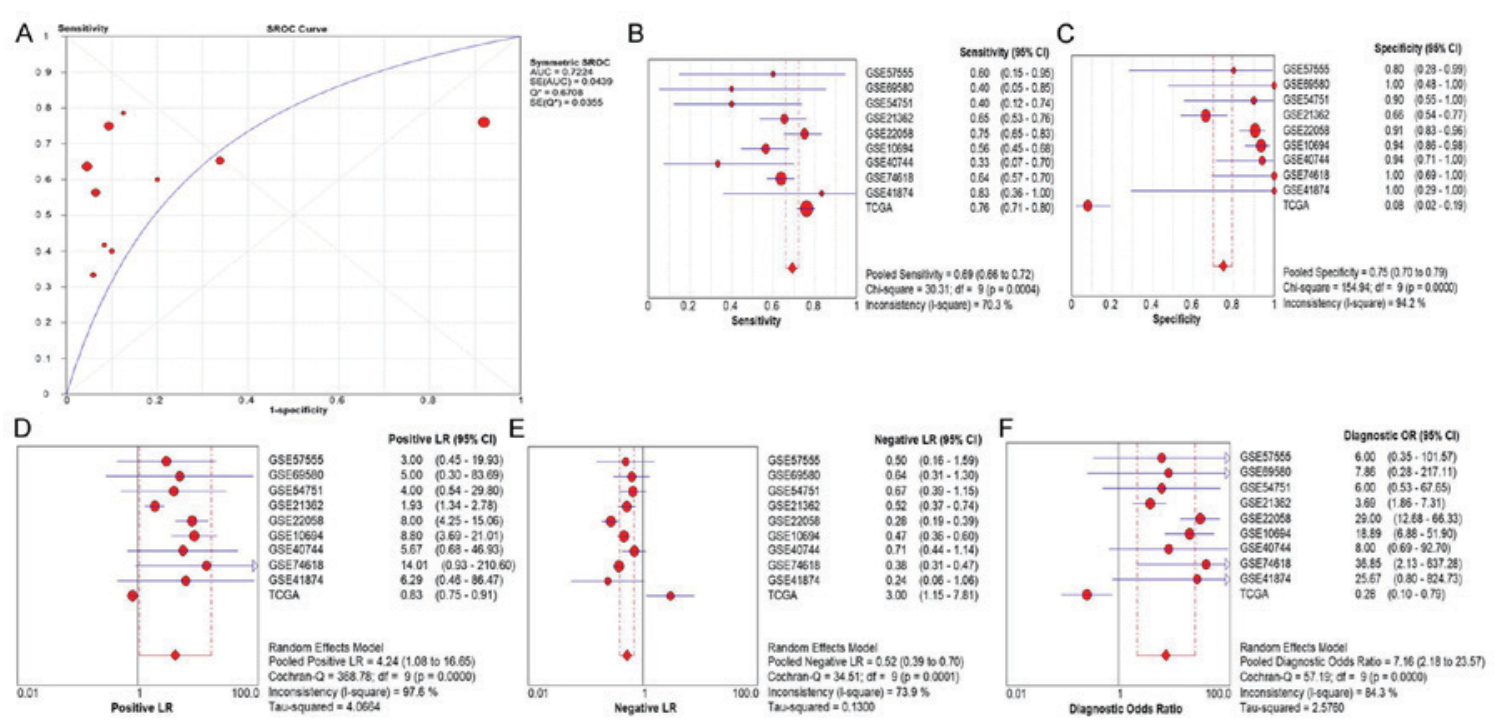

Figure 8. The pooled (A) SROC curve, (B) SEN, (C) SPE, (D) PLR, (E) NLR and (F) DOR of miR-15b-5p in adjacent non-cancerous hepatic tissues. SROC, summarized receiver operating characteristic; SEN, sensitivity; SPE, specificity; PLR, positive likelihood ratio; NLR, negative likelihood ratio; DOR, diagnostic odds ratio.

(Table IV; Figs. 14B and 15). A PPI network of the 225 genes was constructed in the present study with 224 nodes and 221 edges. In the network, ACACB, RIPK4, MAP2K1, TLR4, and IGF1 were identified as the hub target genes of miR-15b-5p due to the highest significance (Figs. 16-18). In the respect to the bioinformatics analyses with these hub genes, the 'activation of MAPK activity' $\left(\mathrm{P}=2.39 \times 10^{-4}\right)$, 'ATP binding' $\left(\mathrm{P}=4.17 \times 10^{-2}\right)$ were regarded as the most significant GO categories. The pathways of 'HIF-1 signaling pathway' $\left(\mathrm{P}=5.92 \times 10^{-4}\right)$, 'Proteoglycans in cancer' $\left(\mathrm{P}=2.45 \times 10^{-3}\right)$ and 'PI3K-Akt signaling pathway' $\left(\mathrm{P}=7.21 \times 10^{-3}\right)$ were considered to be the most significant pathways as assessed by KEGG (Tables V and VI). We also ascertained the downregulation of ACACB, RIPK4, MAP2K1, TLR4 and IGF1 in HCC tissues via TCGA data. To verify the possibility that these hub genes were targeted by miR-15b-5p, we further revealed the protein levels of ACACB, RIPK4, MAP2K1, TLR4 and IGF1 in HCC tissues and normal tissues. As revealed in Fig. 19, ACACB had medium staining and moderate intensity in cytoplasmic/membranous normal liver tissues. MAP2K1, TLR4 and IGF1 exhibited low staining and weaker intensity in cytoplasmic/membranous normal liver tissues. In addition, all these hub genes had a lower staining and weaker intensity in HCC tissues. These findings warrant further validation, as limited sample size is provided by the HPA.

\section{Discussion}

In the present study, the results of the investigation demonstrated an overall moderate test performance of miR-15b-5p with respect to its clinical value. The summary sensitivity of the plasma/serum miR-15b-5p (81\%) revealed superiority 


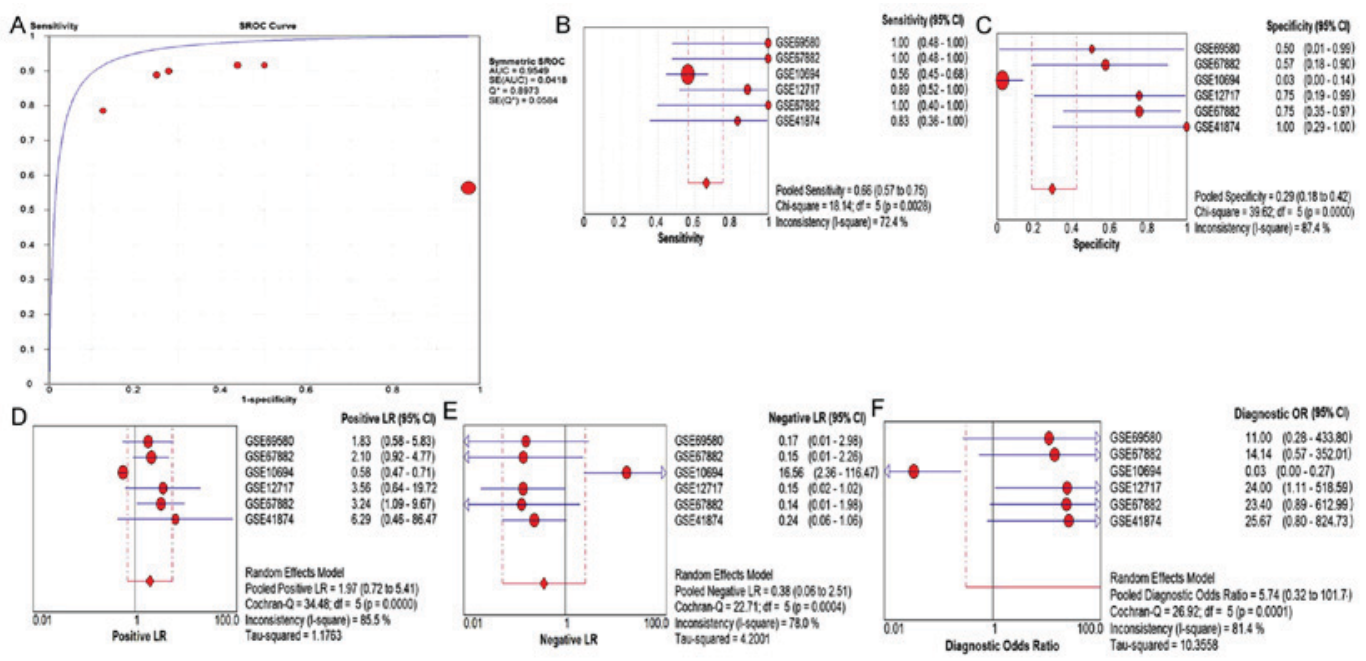

Figure 9. The pooled (A) SROC curve, (B) SEN, (C) SPE, (D) PLR, (E) NLR and (F) DOR of miR-15b-5p in HCC and $\mathrm{HBV}^{+}$or $\mathrm{HCV}^{+}$patients. SROC, summarized receiver operating characteristic; SEN, sensitivity; SPE, specificity; PLR, positive likelihood ratio; NLR, negative likelihood ratio; DOR, diagnostic odds ratio; HCC, hepatocellular carcinoma.

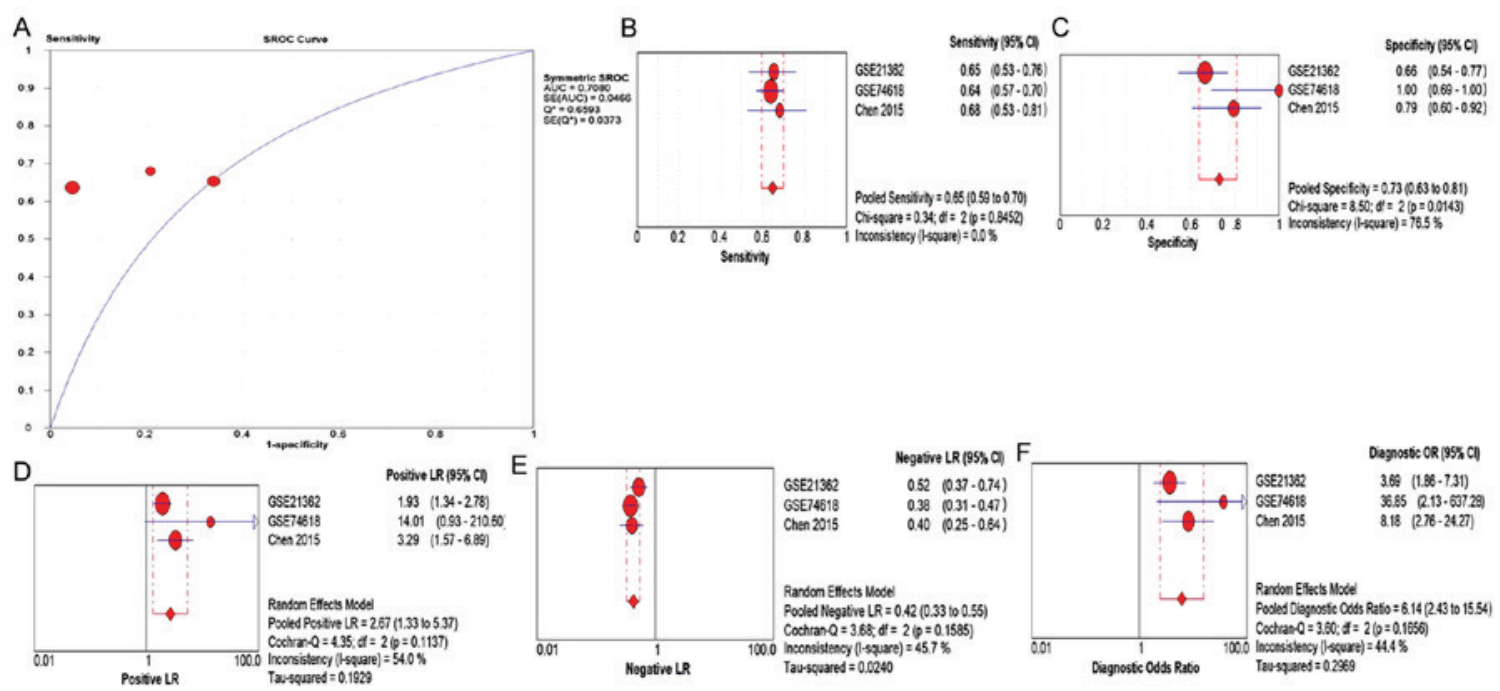

Figure 10. The pooled (A) SROC curve, (B) SEN, (C) SPE, (D) PLR, (E) NLR and (F) DOR of miR-15b-5p in liver cirrhosis. SROC, summarized receiver operating characteristic; SEN, sensitivity; SPE, specificity; PLR, positive likelihood ratio; NLR, negative likelihood ratio; DOR, diagnostic odds ratio.

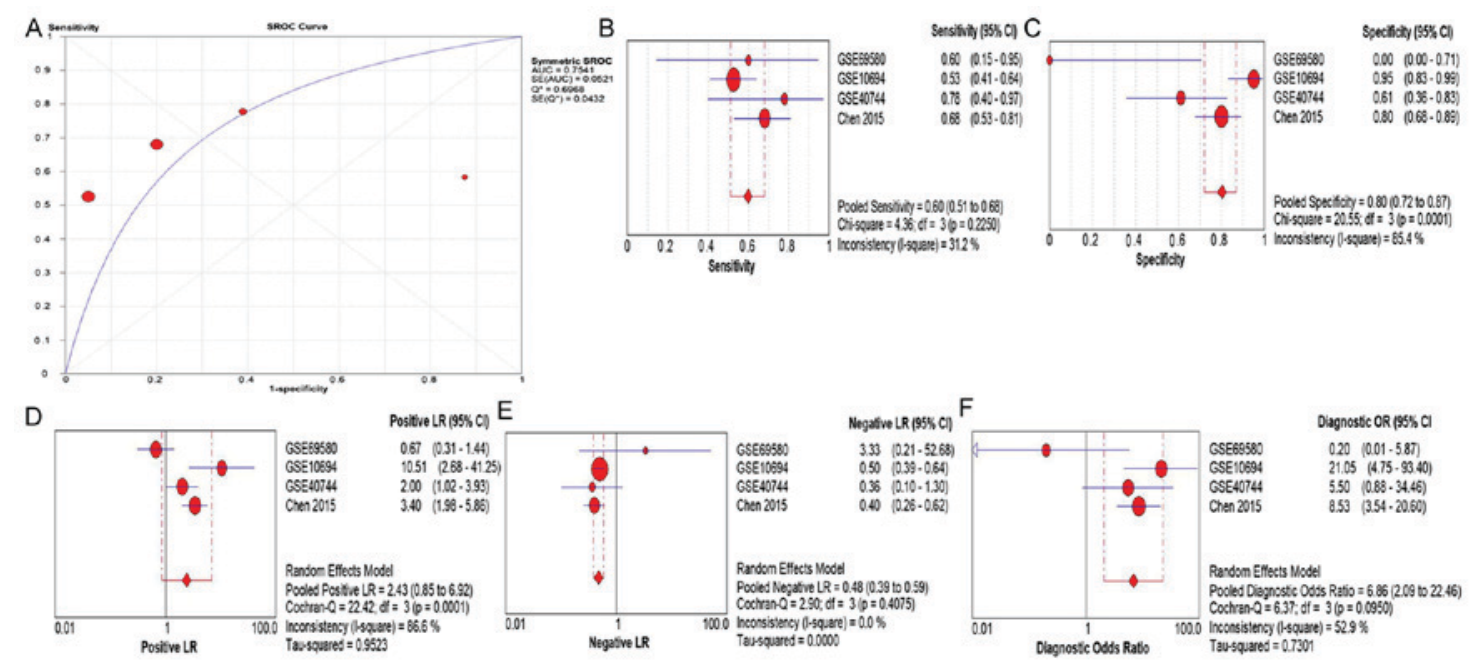

Figure 11. The pooled (A) SROC curve, (B) SEN, (C) SPE, (D) PLR, (E) NLR and (F) DOR of miR-15b-5p in HBV $\mathrm{H}^{+} \mathrm{HCV}^{+}$and cirrhosis. SROC, summarized receiver operating characteristic; SEN, sensitivity; SPE, specificity; PLR, positive likelihood ratio; NLR, negative likelihood ratio; DOR, diagnostic odds ratio. 
A

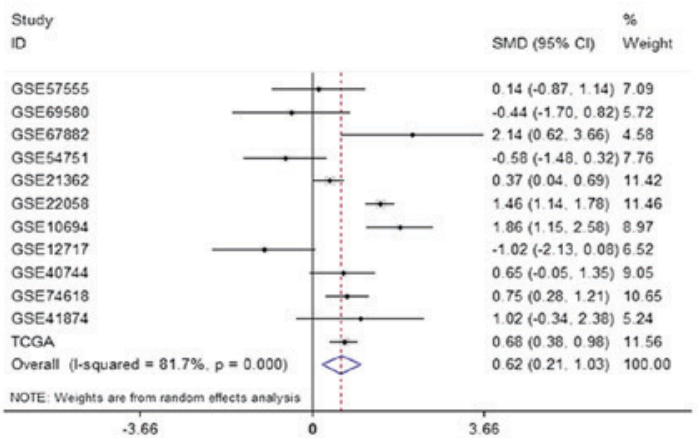

C

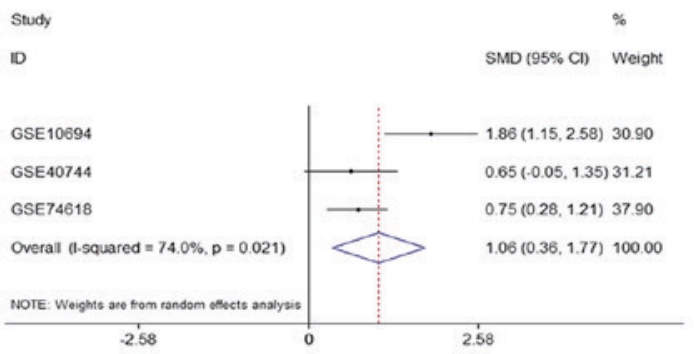

$\mathrm{E}$

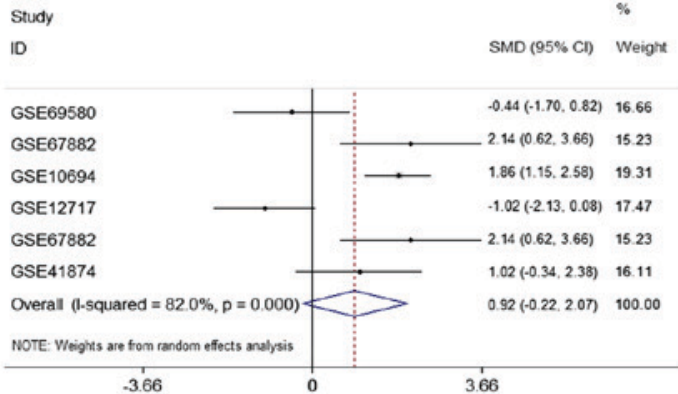

G Study

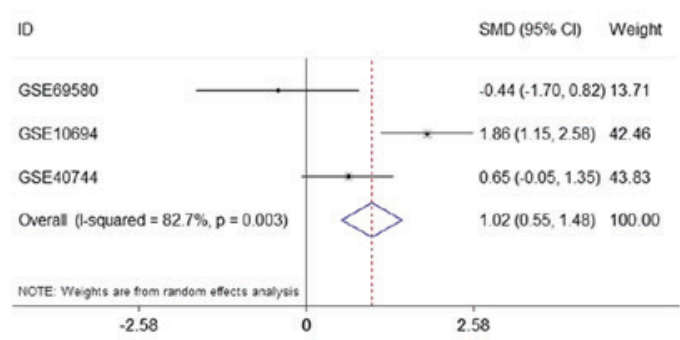

B

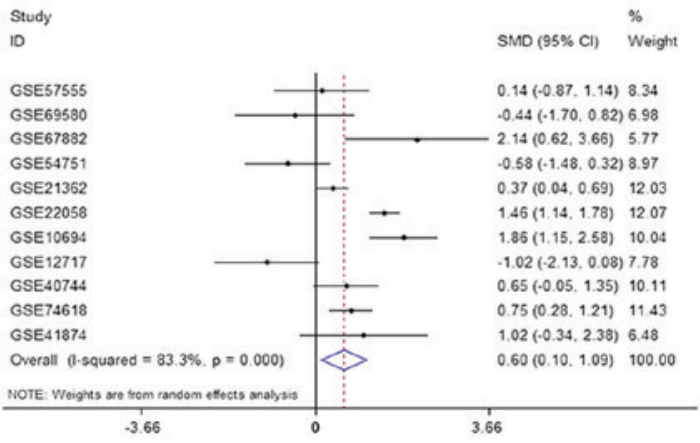

D stud

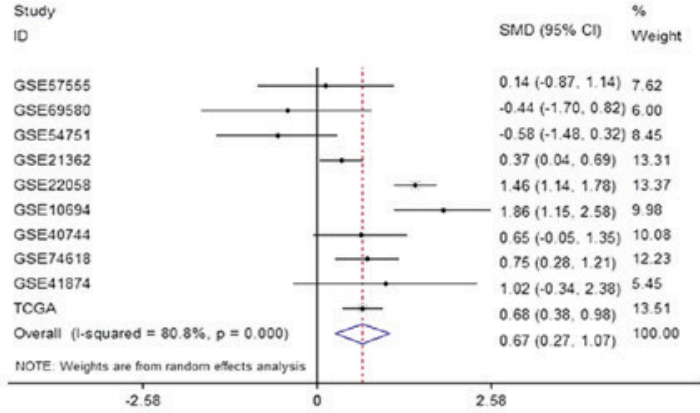

$\mathrm{F}$
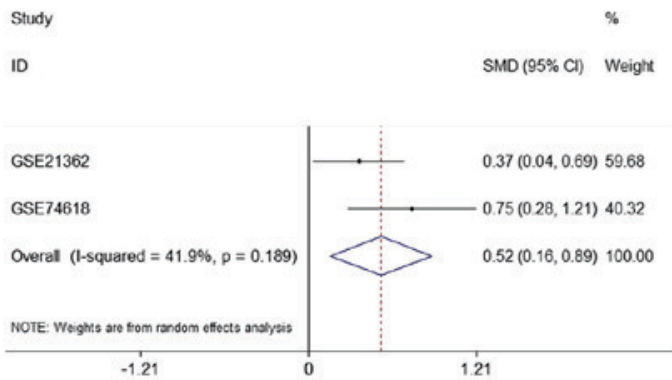

Figure 12. Forest plots of studies calculating the SMD of miR-15b-5p expression in (A) the HCC group, (B) tissues, (C) healthy people, (D) adjacent non-cancerous hepatic tissues, (E) $\mathrm{HCC}$ and $\mathrm{HBV}^{+}$or $\mathrm{HCV}^{+}$patients, (F) liver cirrhosis and (G) $\mathrm{HBV}^{+} / \mathrm{HCV}^{+}$and cirrhosis compared with the control group based on the random effect model. SMD, standard mean difference; HCC, hepatocellular carcinoma.

compared to AFP despite an overall sensitivity of less than $60 \%$. The AUC value was 0.83 in serum and 0.82 in tissue. Data comparing HCC with non-HCC tissue in the same liver or others controls indicated different factors. In our study, detection of miR-15b-5p in serum/plasma sample had a more favorable diagnostic accuracy than that in tissues. The results also revealed via SMD analysis, that the miR-15b-5p expression level in HCC was markedly overexpressed when compared to non-HCC tissues samples. In detail, the random-effects model was used for the pooled SMD of miR-15b-5p to resolve the problem of heterogeneity. Furthermore, to decrease the heterogeneity, we also performed subgroup analyses with both SMD and sROC methods. The subgroups included sample sources (tissues and serum/plasma) and control types (healthy controls, adjacent non-cancerous hepatic tissues, $\mathrm{HBV}^{+}$or $\mathrm{HCV}^{+}$tissues, liver cirrhosis tissues, and those combining $\mathrm{HBV}^{+} / \mathrm{HCV}^{+}$and cirrhosis), which revealed that miR-15b-5p may be a prospective biomarker to distinguish HCC patients from healthy people. Numerous HCC patients have a background of liver cirrhosis and chronic HBV and/or HCV. Whether circulating miR-15b-5p can be used to differentiate HCC from benign hepatic lesions has been studied (16). Furthermore, our analysis of the studies indicated that the summary sensitivity and specificity of miR-15b-5p for distinguishing HCC from chronic HBV and/or HCV as well as liver cirrhosis were 60 and $80 \%$, respectively, indicating a potential value that was worth exploring. It is regrettable 

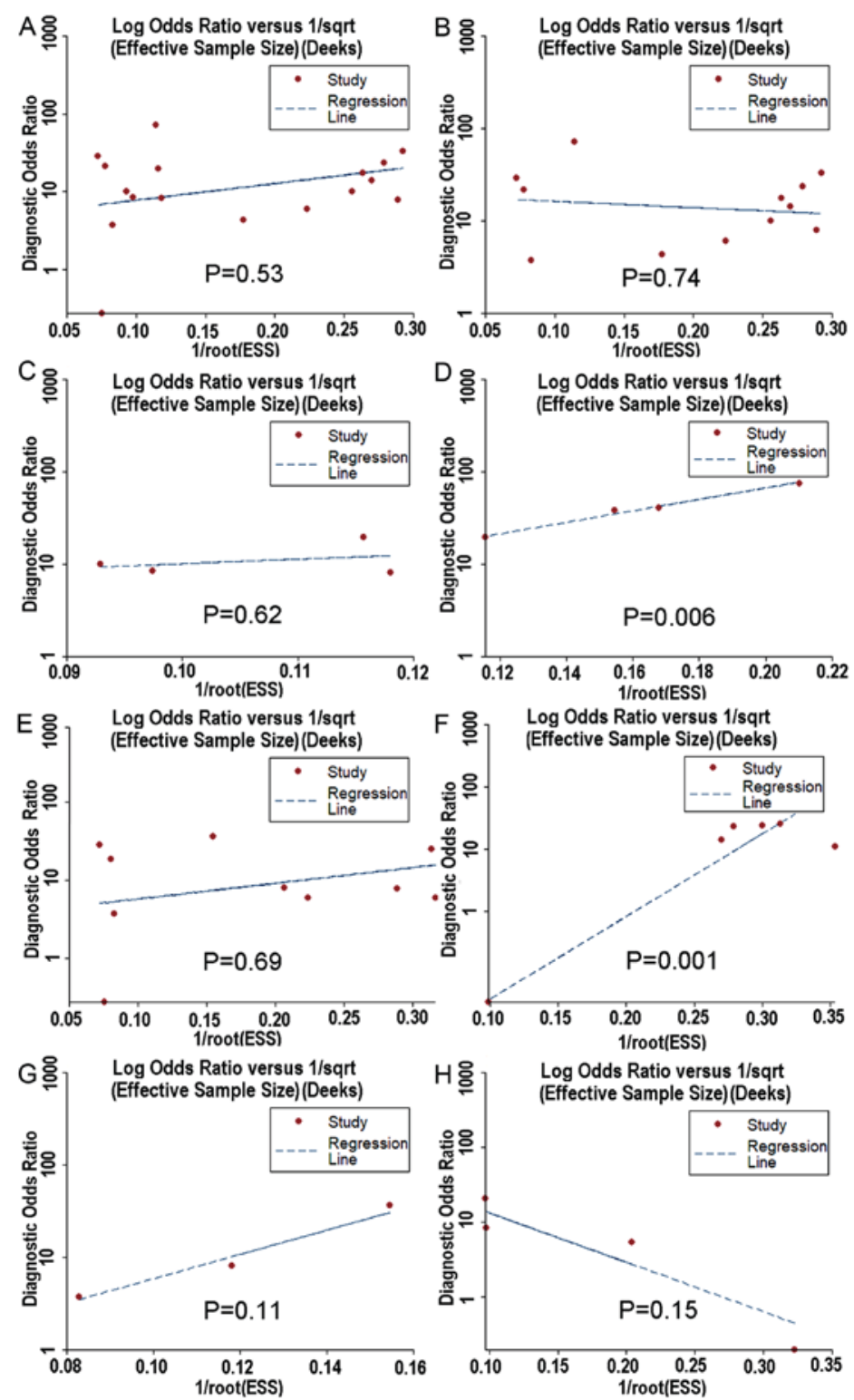

Figure 13. Symmetrical Deek's funnel plot are used to estimate the publication bias between the different subgroups. (A) HCC group, (B) tissues, (C) serum/plasma, (D) healthy people, (E) adjacent non-cancerous hepatic tissues, (F) $\mathrm{HCC}$ and $\mathrm{HBV}^{+}$or $\mathrm{HCV}^{+}$patients, (G) liver cirrhosis and (H) $\mathrm{HBV}^{+} / \mathrm{HCV}^{+}$and cirrhosis.

that there is no evidence that miR-15b-5p is related to the progression of HCC, which is worthy of further study.

Some studies have revealed that circulating miRNAs can be potential markers for HCC determination. These miRNAs have been reported to be deregulated in cirrhosis and during development of hepatic malignancy. Abdalla et al summarized the tested diagnostic accuracy of miR-618 and miR-650 in HCC which were revealed to be 0.71 and 0.70 , and may be of great value for the early diagnosis of HCC (28). Tan et al reported that serum miR-206, miR-141-3p, miR-433-3p, miR-1228-5p, miR-199a-5p, miR-122-5p, miR-192-5p and miR-26a-5p were potential circulating markers for the diagnosis of HCC with AUC from 0.53-0.73 (29). In the current study, compared with the non-neoplastic controls, the miR-15b-5p levels in HCC revealed a favorable diagnostic value with a pooled AUC of 0.81 which indicated that miR-15b-5p may be a prospective biomarker to distinguish HCC patients from non-HCC controls. Our studies on miR-15b-5p in HCC are limited and the role of miR-15b-5p remains largely unknown. The main limitation of this study was that the sample size was small and present findings should be validated in trials with more cases.

With regards to the existing related literature, there were 3 studies that addressed the clinical potential of miR-15b-5p. Hung et al (14) first indicated that miR-15b-5p could be utilized for the early detection of HCC. However, no explicit diagnostic test was conducted in this study. The study of Liu et al (15) noted that binding of miR-130b and miR-15b-5p could improve the accuracy of HCC diagnosis based on expression data collected from HCC patients, HBV carriers and healthy controls. Chen et al (16) designed three subgroups including $\mathrm{HCC}$ patients, cirrhosis patients and healthy individuals to evaluate the clinical potential of miR-15b-5p. Only two genes: OIP5 and Rab1A have been revealed to be the direct targets of miR-15b-5p in HCC, however, the existing 

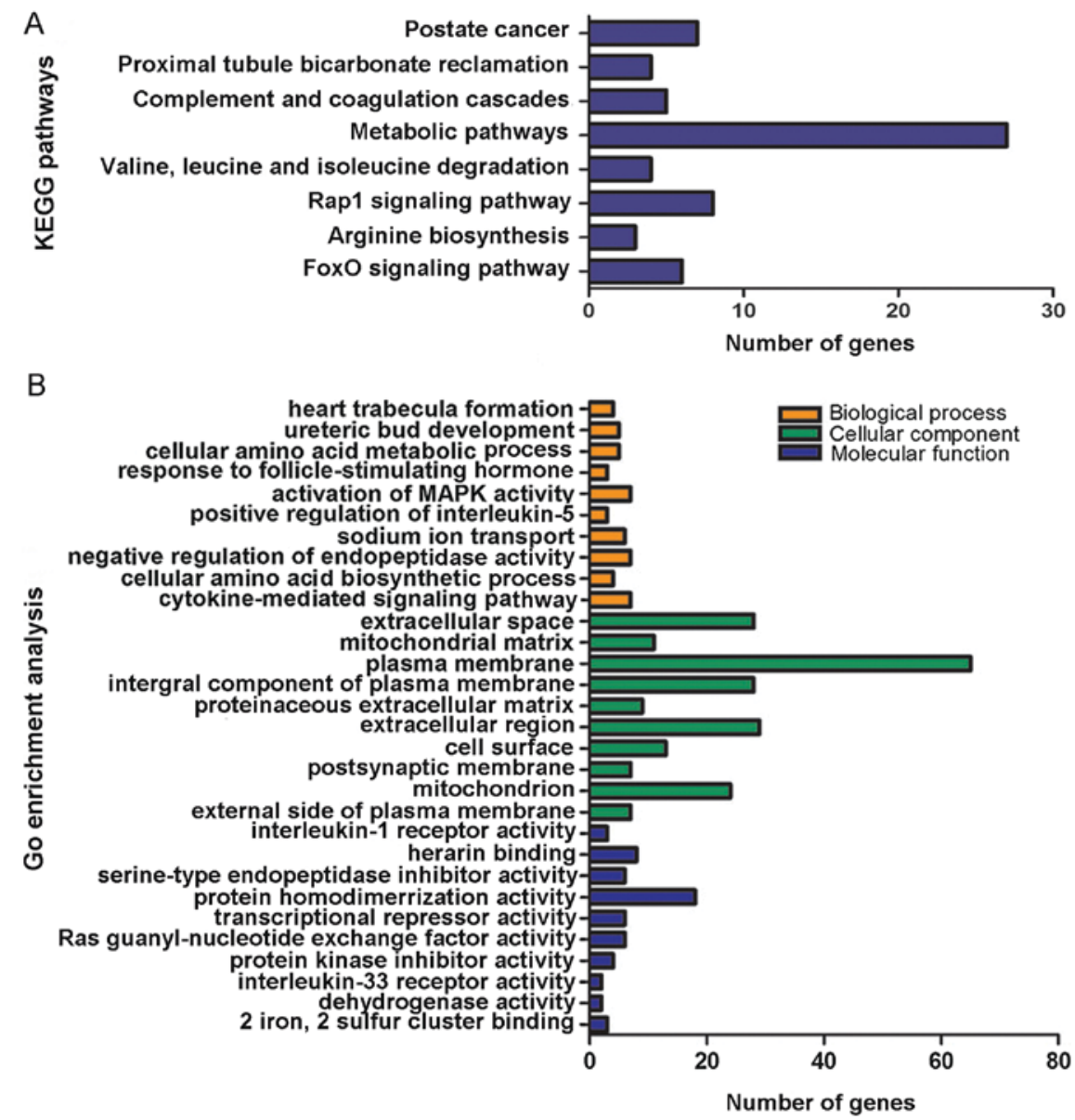

Figure 14. KEGG and GO analysis of miR-15b-5p target genes. (A) KEGG analysis of the miR-15b-5p target genes. (B) GO functional annotation according to the putative target genes of miR-15b-5p in HCC. KEGG, Kyoto Encyclopedia of Genes and Genomes; GO, Gene ontology; HCC, hepatocellular carcinoma.

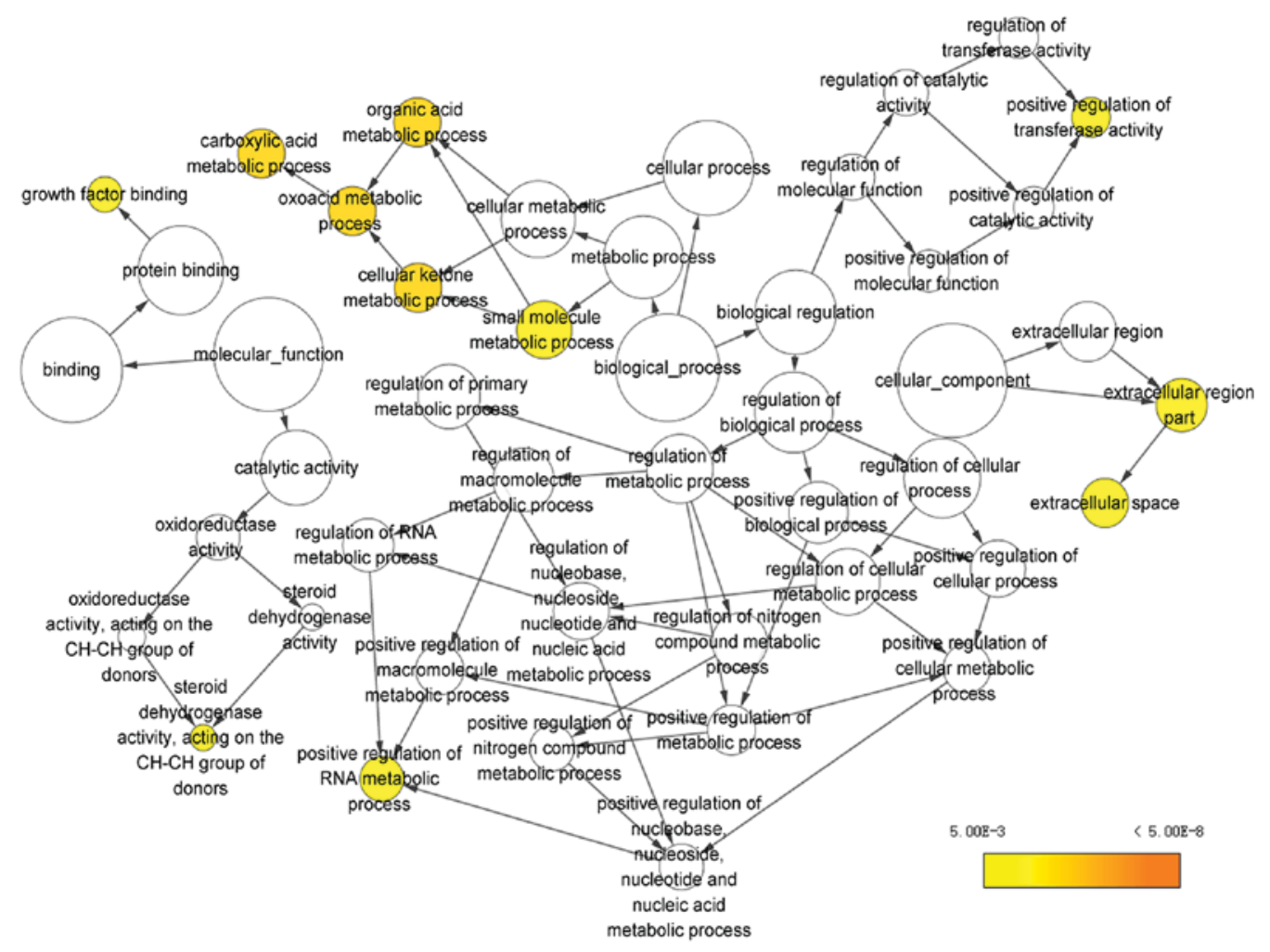

Figure 15. GO functional annotation according to the putative target genes of miR-15b-5p in HCC using the BiNGO plugin in Cytoscape. GO, Gene ontology; HCC, hepatocellular carcinoma. 


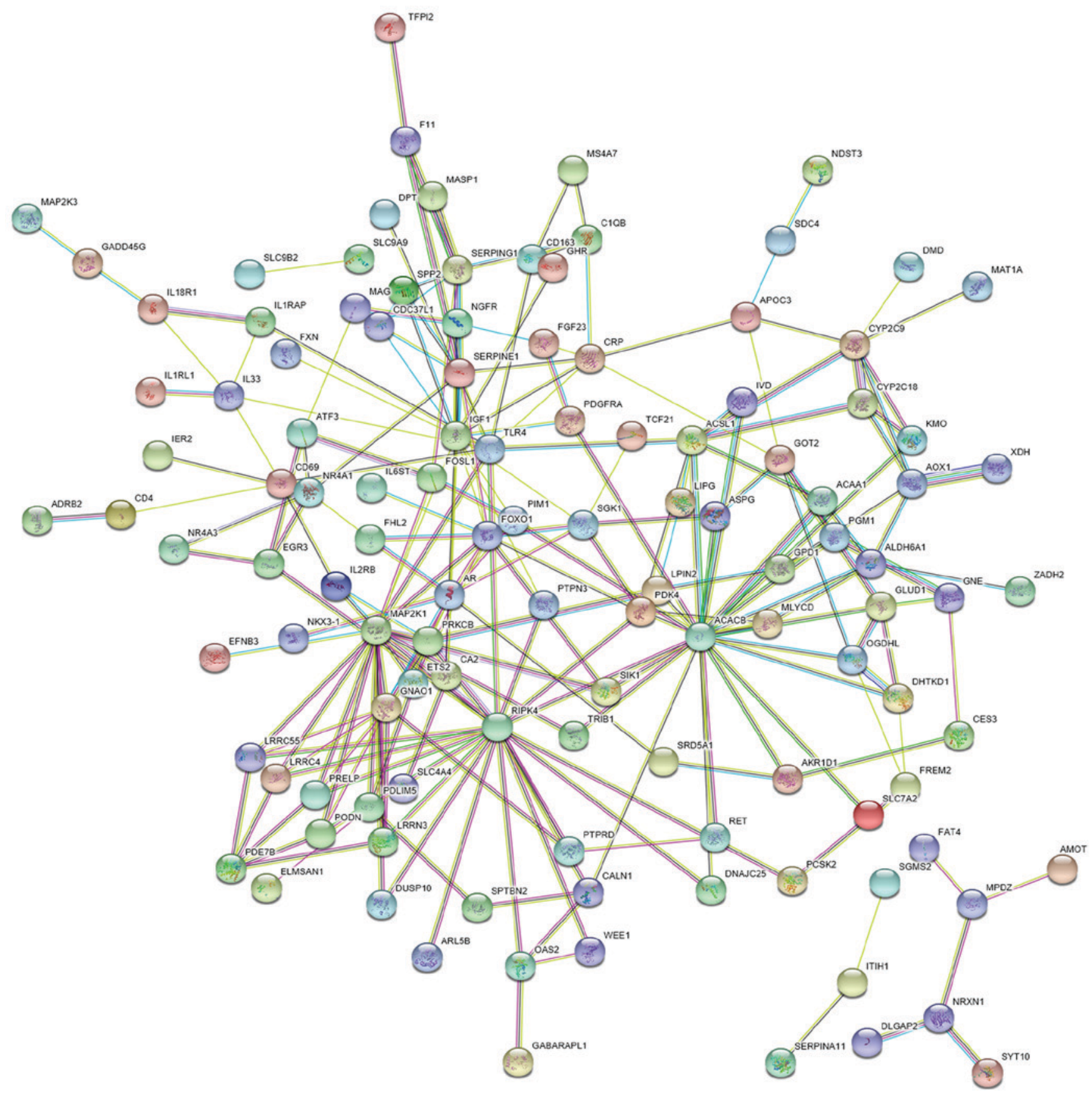

Figure 16. PPI network of the target genes of miR-15b-5p. A total of 224 nodes and 221 edges composed the network. The graph hides the disconnected nodes in the network and the top 5 hub genes with the most interaction lines in PPI analysis. PPI, protein-protein interactions.

studies solely concentrated on the performance of circulating miR-15b-5p. Therefore, we collected expression profiles in hepatic tissues to broaden the spectrum of research and carried out a comprehensive analysis.

In bioinformatics analyses, KEGG analysis highlighted the insulin signaling pathway, which was connected with other enriched pathways in our analysis. Insulin can induce phosphorylation of the insulin receptor substrate (IRS), thus allowing IRS to interact with the PI3K/Akt signaling pathway and MAPK signaling pathway, which are involved in biological mechanisms, such as glycogen synthesis, cell glucose intake, protein synthesis and gene transcription (30). In HCC, aberrantly elevated insulin receptor and IRS-1 were demonstrated to have a correlation with tumorigenesis contributing to precancerous liver glycogenosis and hepatocellular proliferation (31-33). Tanaka et al also reported that IRS-1 could impede transforming growth factor $\beta 1$-induced cell apoptosis, which may increase the risk of HCC (34). In association with IRS-1, the PI3K/Akt signaling pathway and MAPK signaling pathway were also considered to play roles in the molecular mechanism of HCC, and the joint effect of these pathways was also investigated by researchers. Liang et al (35) reported that aconitum coreanum polysaccharide inhibited the expression of pituitary tumor transforming gene 1, an oncogene, by suppressing the PI3K/Akt signaling pathway and upregulating the MAPK signaling pathway. A study from Gedaly et al (36) also discovered that targeting the PI3K/Akt signaling pathway and MAPK signaling pathway could attenuate the proliferation 

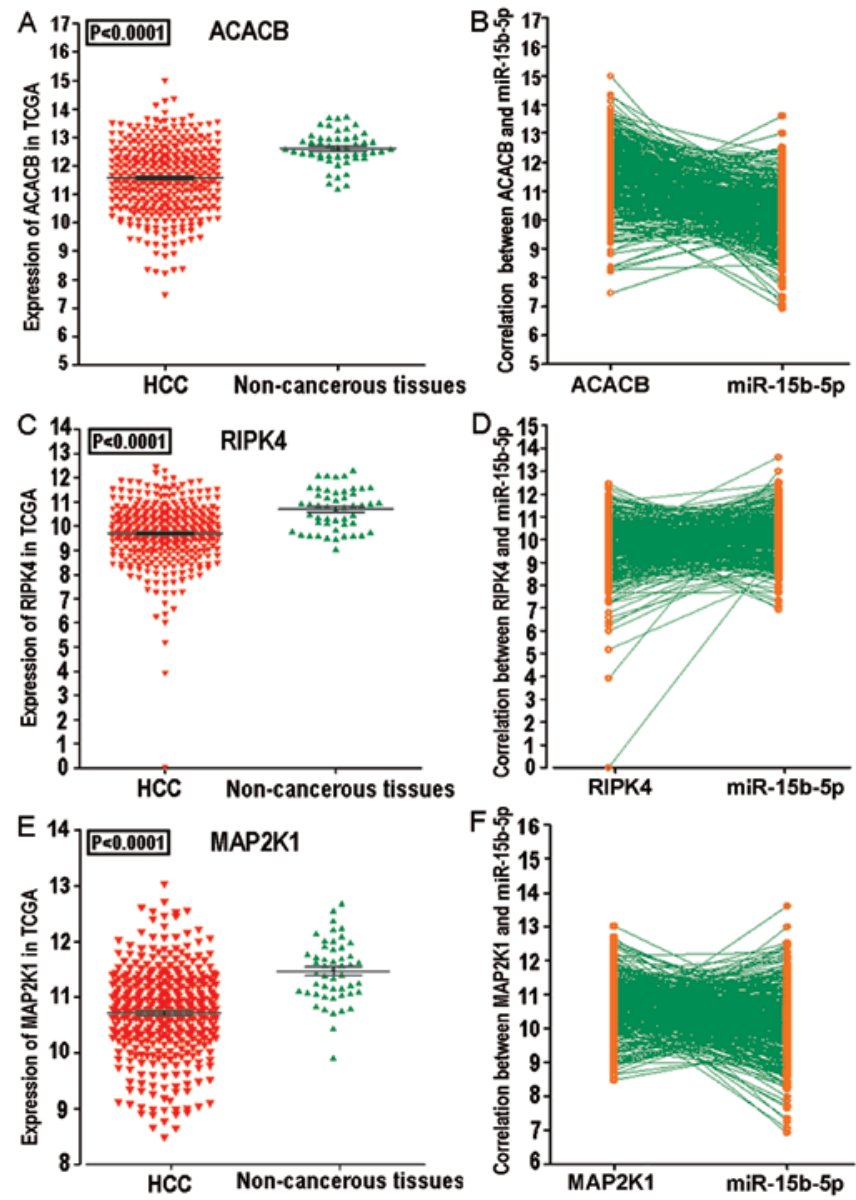

Figure 17. mRNA expression levels of three hub target genes of miR-15b-5p in HCC. The mRNA expression levels of these targets were calculated by TCGA. (A, C and E) The mRNA levels were significantly downregulated in HCC tissues compared to those in non-cancerous liver tissues. (B, D and F) The relevant relationship between the expression of targets and miR-15b-5p. HCC, hepatocellular carcinoma; TCGA, The Cancer Genome Atlas.
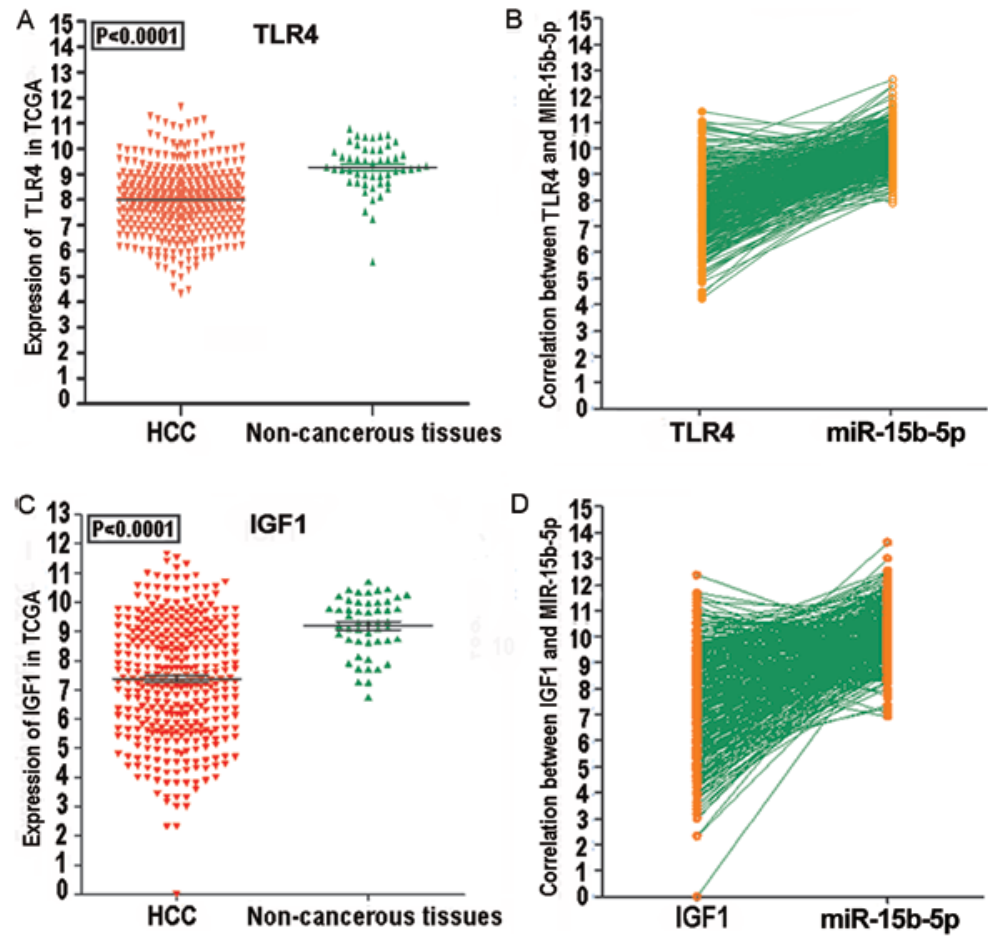

Figure 18. mRNA expression levels of two hub target genes of miR-15b-5p in HCC. The mRNA expression levels of these targets were calculated by TCGA. (A and C) The mRNA levels were significantly downregulated in HCC tissues compared to those in non-cancerous liver tissues. (B and D) The relevant relationship between the expression of targets and miR-15b-5p. HCC, hepatocellular carcinoma; TCGA, The Cancer Genome Atlas. 
A

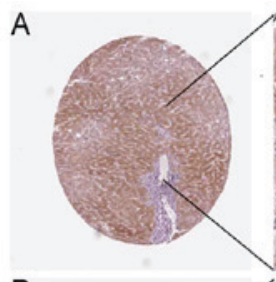

B

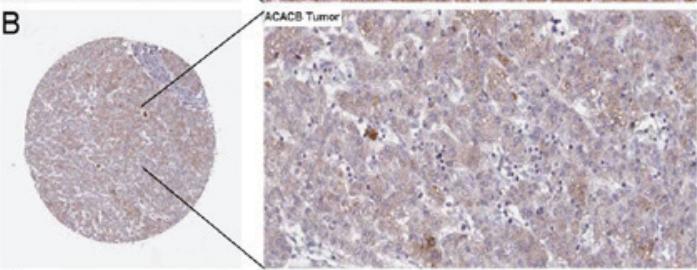

E

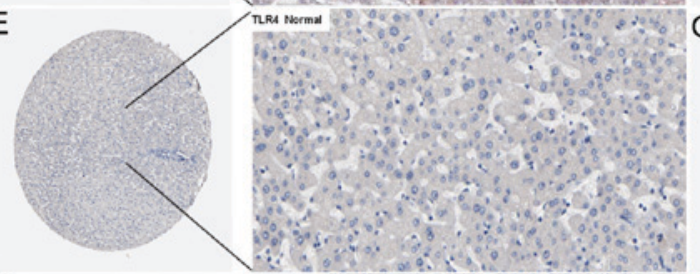

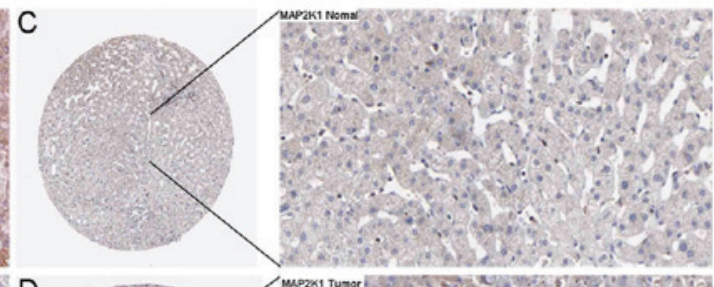

D

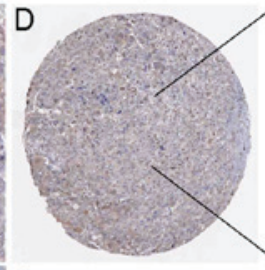

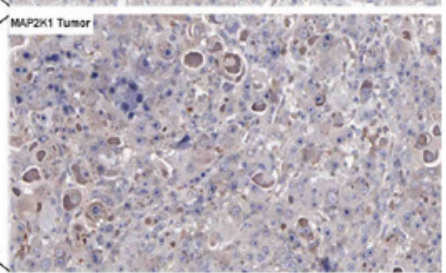

G
F

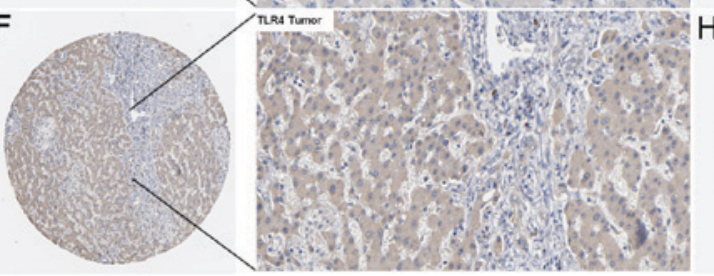

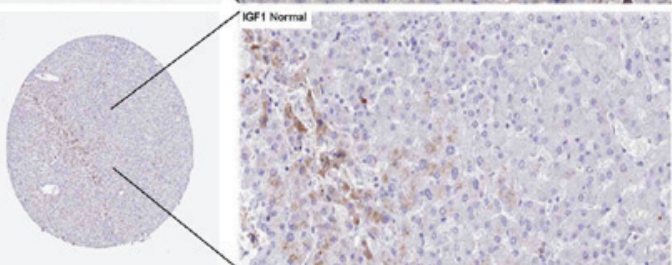

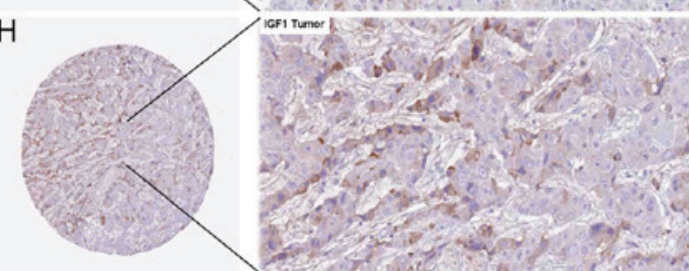

Figure 19. Immunohistochemical staining of four hub target genes of miR-15b-5p in HCC. IHC of ACACB, MAP2K1, TLR4 and IGF1 in normal liver tissues and HCC tissues were provided by the HPA. ACACB expression in (A) normal and (B) HCC tissues (antibody HPA006554). MAP2K1 expression in (C) normal and (D) HCC tissues (antibody CAB003834). TLR4 expression in (E) normal and (F) HCC tissues (antibody CAB004025). IGF1 expression in (G) normal and (H) HCC tissues (antibody HPA048946). ACACB had medium staining and moderate intensity in cytoplasmic/membranous normal liver tissues. MAP2K1, TLR4 and IGF1 had low staining and weaker intensity in cytoplasmic/membranous normal liver tissues. By contrast, lower staining and weaker intensity of these hub genes was detected in HCC tissues. Immunohistochemical images were obtained using a microscope (magnification, $\mathrm{x} 40$ or $\mathrm{x} 400$ ). IHC, immunohistochemistry; HPA, Human Protein Atlas; HCC, hepatocellular carcinoma.

of HCC cells. Moreover, these two signaling pathways were also revealed to have independent correlations with HCC cell growth $(37,38)$ apoptosis $(39,40)$ migration, and invasion, as well as adhesion (41-43), which affects the tumorigenesis and progression of HCC $(44,45)$ via various genes. Therefore, we concluded that miR-15b-5p may participate in hepatocellular carcinogenesis via diverse pathways.

However, certain limitations still exist in our study. Significant heterogeneity was considered in our meta-analysis. The sample types, study design, population, sample size and some of the clinical characteristics may contribute to the increased heterogeneity. Furthermore, overexpression of miR-15b-5p has been previously reported in other malignancies, such as colorectal cancer (46), endometrial endometrioid adenocarcinoma (47) and non-small cell lung cancer (48). In summary, we confirmed that increased miR-15b-5p may not be particularly connected with HCC itself, however it affects the progression of different cancers. For this reason, in daily clinical practice, miR-15b-5p alone may not play an essential role for $\mathrm{HCC}$, however if it is used in combination with other markers, this biomarker may improve test performance.

To conclude, a high miR-15b-5p level may be one the probable causes of HCC tumorigenesis, however, it could also be the consequence after HCC has already occurred, which warrants further investigation. Based on the marked upregulation of the level of miR-15b-5p in HCC, its potential clinical value is anticipated, which also requires practical validation. Notably, the prospective role and signaling pathways of miR-15b-5p have been revealed by in silico methods only, and thus, the specific mechanism of miR-15b-5p in HCC requires further study.

\section{Acknowledgements}

Not applicable.

\section{Funding}

The present study was supported by a fund from the National Natural Science Foundation of China (NSFC81560386).

\section{Availability of data and materials}

The datasets used during the present study are available from the corresponding author upon reasonable request.

\section{Authors' contributions}

WYP collected the public data and drafted the present study. JHZ and PPW performed the statistical analysis and constructed enrichment pathways. DYW and JYW re-analyzed 
the data of the results and drafted the section of the results, including writing and modifying tables and figures. GC and ZBF participated in the design and revision of the study. All authors read and approved the final manuscript

\section{Ethics approval and consent to participate}

Not applicable.

\section{Patient consent for publication}

Not applicable.

\section{Competing interests}

The authors declare that they have no competing interests.

\section{References}

1. Bosetti C, Turati F and La Vecchia C: Hepatocellular carcinoma epidemiology. Best Pract Res Clin Gastroenterol 28: 753-770, 2014.

2. Schwartz M, Roayaie S and Konstadoulakis M: Strategies for the management of hepatocellular carcinoma. Nat Clin Pract Oncol 4: 424-432, 2007.

3. Trevisani F, Cantarini MC, Wands JR and Bernardi M: Recent advances in the natural history of hepatocellular carcinoma. Carcinogenesis 29: 1299-1305, 2008.

4. Daniele B, Bencivenga A, Megna AS and Tinessa V: Alpha-fetoprotein and ultrasonography screening for hepatocellular carcinoma. Gastroenterology 127 (5 Suppl 1): S108-S112, 2004.

5. Li JJ, Luo J, Lu JN, Liang XN, Luo YH, Liu YR, Yang J, Ding H, Qin GH, Yang LH, et al: Relationship between TRAF6 and deterioration of HCC: An immunohistochemical and in vitro study. Cancer Cell Int 16: 76, 2016.

6. Luo Y, Ren F, Liu Y, Shi Z, Tan Z, Xiong H, Dang Y and Chen G: Clinicopathological and prognostic significance of high Ki-67 labeling index in hepatocellular carcinoma patients: A meta-analysis. Int J Clin Exp Med 8: 10235-10247, 2015.

7. Huang Z, Huang L, Shen S, Li J, Lu H, Mo W, Dang Y, Luo D, Chen $\mathrm{G}$ and Feng Z: Sp1 cooperates with Sp3 to upregulate MALAT1 expression in human hepatocellular carcinoma. Oncol Rep 34: 2403-2412, 2015.

8. Zhang X, Tang W, Li R, He R, Gan T, Luo Y, Chen G and Rong M: Downregulation of microRNA-132 indicates progression in hepatocellular carcinoma. Exp Ther Med 12: 2095-2101, 2016.

9. Huang WT, Wang HL, Yang H, Ren FH, Luo YH, Huang CQ, Liang YY, Liang HW, Chen G and Dang YW: Lower expressed miR-198 and its potential targets in hepatocellular carcinoma: A clinicopathological and in silico study. Onco Targets Ther 9: 5163-5180, 2016

10. Zhang X, Tang W, Chen G, Ren F, Liang H, Dang Y and Rong M: An encapsulation of gene signatures for hepatocellular carcinoma, MicroRNA-132 predicted target genes and the corresponding overlaps. PLoS One 11: e0159498, 2016.

11. He R, Yang L, Lin X, Chen X, Lin X, Wei F, Liang X, Luo Y, Wu Y, Gan T, et al: MiR-30a-5p suppresses cell growth and enhances apoptosis of hepatocellular carcinoma cells via targeting AEG-1. Int J Clin Exp Pathol 8: 15632-15641, 2015.

12. Liu Y, Ren F, Luo Y, Rong M, Chen G and Dang Y: Down-regulation of MiR-193a-3p dictates deterioration of HCC: A clinical real-time qRT-PCR study. Med Sci Monit 21: 2352-2360, 2015.

13. Huang YH, Lin KH, Chen HC, Chang ML, Hsu CW, Lai MW, Chen TC, Lee WC, Tseng YH and Yeh CT: Identification of postoperative prognostic microRNA predictors in hepatocellular carcinoma. PLoS One 7: e37188, 2012.

14. Hung $\mathrm{CH}, \mathrm{Hu} \mathrm{TH}$, Lu SN, Kuo FY, Chen $\mathrm{CH}$, Wang JH, Huang CM, Lee CM, Lin CY, Yen YH and Chiu YC: Circulating microRNAs as biomarkers for diagnosis of early hepatocellular carcinoma associated with hepatitis B virus. Int J Cancer 138: 714-720, 2016.
15. Liu AM, Yao TJ, Wang W, Wong KF, Lee NP, Fan ST, Poon RT, Gao C and Luk JM: Circulating miR-15b and miR-130b in serum as potential markers for detecting hepatocellular carcinoma: A retrospective cohort study. BMJ Open 2: e000825, 2012.

16. Chen Y, Chen J, Liu Y, Li S and Huang P: Plasma miR-15b-5p, miR-338-5p, and miR-764 as biomarkers for hepatocellular carcinoma. Med Sci Monit 21: 1864-1871, 2015.

17. Zhang Y, Dang YW, Wang X, Yang X, Zhang R, Lv ZL and Chen G: Comprehensive analysis of long non-coding RNA PVT1 gene interaction regulatory network in hepatocellular carcinoma using gene microarray and bioinformatics. Am J Transl Res 9: 3904-3917, 2017.

18. Zhang Y, He RQ, Dang YW, Zhang XL, Wang X, Huang SN Huang WT, Jiang MT, Gan XN, Xie Y, et al: Comprehensive analysis of the long noncoding RNA HOXA11-AS gene interaction regulatory network in NSCLC cells. Cancer Cell Int 16: 89, 2016.

19. Kew MC: Epidemiology of chronic hepatitis B virus infection, hepatocellular carcinoma, and hepatitis B virus-induced hepatocellular carcinoma. Pathol Biol (Paris) 58: 273-277, 2010.

20. Murakami Y, Kubo S, Tamori A, Itami S, Kawamura E, Iwaisako K, Ikeda K, Kawada N, Ochiya T and Taguchi YH: Comprehensive analysis of transcriptome and metabolome analysis in Intrahepatic Cholangiocarcinoma and Hepatocellular Carcinoma. Sci Rep 5: 16294, 2015.

21. Shen J, LeFave C, Sirosh I, Siegel AB, Tycko B and Santella RM: Integrative epigenomic and genomic filtering for methylation markers in hepatocellular carcinomas. BMC Med Genomics 8: $28,2015$.

22. Sato F, Hatano E, Kitamura K, Myomoto A, Fujiwara T, Takizawa S, Tsuchiya S, Tsujimoto G, Uemoto S and Shimizu K: MicroRNA profile predicts recurrence after resection in patients with hepatocellular carcinoma within the Milan Criteria. PLoS One 6: e16435, 2011.

23. Burchard J, Zhang C, Liu AM, Poon RT, Lee NP, Wong KF, Sham PC,Lam BY, Ferguson MD, Tokiwa G, et al: microRNA-122 as a regulator of mitochondrial metabolic gene network in hepatocellular carcinoma. Mol Syst Biol 6: 402, 2010.

24. Li W, Xie L, He X, Li J, Tu K, Wei L, Wu J, Guo Y, Ma X, Zhang $\mathrm{P}$, et al: Diagnostic and prognostic implications of microRNAs in human hepatocellular carcinoma. Int J Cancer 123: 1616-1622, 2008.

25. Su H, Yang JR, Xu T, Huang J, Xu L, Yuan Y and Zhuang SM: MicroRNA-101, down-regulated in hepatocellular carcinoma, promotes apoptosis and suppresses tumorigenicity. Cancer Res 69: 1135-1142, 2009.

26. Diaz G, Melis M, Tice A, Kleiner DE, Mishra L, Zamboni F and Farci P: Identification of microRNAs specifically expressed in hepatitis $\mathrm{C}$ virus-associated hepatocellular carcinoma. Int J Cancer 133: 816-824, 2013.

27. Martinez-Quetglas I, Pinyol R, Dauch D, Torrecilla S, Tovar V, Moeini A, Alsinet C, Portela A, Rodriguez-Carunchio L, Solé M, et al: IGF2 Is Up-regulated by epigenetic mechanisms in hepatocellular carcinomas and is an actionable oncogene product in experimental models. Gastroenterology 151: 1192-1205, 2016.

28. Abdalla MA and Haj-Ahmad Y: Promising candidate urinary MicroRNA biomarkers for the early detection of hepatocellular carcinoma among high-risk Hepatitis $\mathrm{C}$ virus Egyptian patients. J Cancer 3: 19-31, 2012.

29. Tan Y, Ge G, Pan T, Wen D, Chen L, Yu X, Zhou X and Gan J: A serum microRNA panel as potential biomarkers for hepatocellular carcinoma related with hepatitis B virus. PLoS One 9: e107986, 2014.

30. Bevan P: Insulin signalling. J Cell Sci 114: 1429-1430, 2001.

31. Aleem E, Nehrbass D, Klimek F, Mayer D and Bannasch P: Upregulation of the insulin receptor and type I insulin-like growth factor receptor are early events in hepatocarcinogenesis. Toxicol Pathol 39: 524-543, 2011.

32. Nehrbass D, Klimek F and Bannasch P: Overexpression of insulin receptor substrate-1 emerges early in hepatocarcinogenesis and elicits preneoplastic hepatic glycogenosis. Am J Pathol 152: 341-345, 1998.

33. Mohr L, Banerjee K, Kleinschmidt M, Bartolomé Rodriguez MM and Wands JR: Transgenic overexpression of insulin receptor substrate 1 in hepatocytes enhances hepatocellular proliferation in young mice only. Hepatol Res 38: 1233-1240, 2008.

34. Tanaka S and Wands JR: Insulin receptor substrate 1 overexpression in human hepatocellular carcinoma cells prevents transforming growth factor beta1-induced apoptosis. Cancer Res 56: 3391-3394, 1996. 
35. Liang M, Liu J, Ji H, Chen M, Zhao Y, Li S, Zhang X and Li J: A Aconitum coreanum polysaccharide fraction induces apoptosis of hepatocellular carcinoma (HCC) cells via pituitary tumor transforming gene 1 (PTTG1)-mediated suppression of the P13K/Akt and activation of p38 MAPK signaling pathway and displays antitumor activity in vivo. Tumour Biol 36: 7085-7091, 2015.

36. Gedaly R, Angulo P, Hundley J, Daily MF, Chen C and Evers BM: PKI-587 and sorafenib targeting PI3K/AKT/mTOR and Ras/Raf/MAPK pathways synergistically inhibit HCC cell proliferation. J Surg Res 176: 542-548, 2012.

37. Wu R, Duan L, Cui F, Cao J, Xiang Y, Tang Y and Zhou L: S100A9 promotes human hepatocellular carcinoma cell growth and invasion through RAGE-mediated ERK1/2 and p38 MAPK pathways. Exp Cell Res 334: 228-238, 2015.

38. Fang X, Yang D, Luo H, Wu S, Dong W, Xiao J, Yuan S, Ni A, Zhang KJ, Liu XY and Chu L: SNORD126 promotes HCC and CRC cell growth by activating the PI3K-AKT pathway through FGFR2. J Mol Cell Biol 9: 243-255, 2017.

39. Bao H, Liu P, Jiang K, Zhang X, Xie L, Wang Z and Gong P: Huaier polysaccharide induces apoptosis in hepatocellular carcinoma cells through p38 MAPK. Oncol Lett 12: 1058-1066, 2016

40. Jiang S, Wang Q, Feng M, Li J, Guan Z, An D, Dong M, Peng Y, Kuerban $\mathrm{K}$ and Ye L: C2-ceramide enhances sorafenib-induced caspase-dependent apoptosis via PI3K/AKT/mTOR and Erk signaling pathways in HCC cells. Appl Microbiol Biotechnol 101: $1535-1546,2017$

41. Zhu B, Shi S, Ma YG, Fan F and Yao ZZ: Lysophosphatidic acid enhances human hepatocellular carcinoma cell migration, invasion and adhesion through P38 MAPK pathway. Hepatogastroenterology 59: 785-789, 2012.
42. Hsieh YH, Hsieh SC, Lee CH, Yang SF, Cheng CW, Tang MJ, Lin CL, Lin CL and Chou RH: Targeting EMP3 suppresses proliferation and invasion of hepatocellular carcinoma cells through inactivation of PI3K/Akt pathway. Oncotarget 6: 34859-34874, 2015.

43. Zheng Y, Wang X, Wang H, Yan W, Zhang Q and Chang X: Bone morphogenetic protein 2 inhibits hepatocellular carcinoma growth and migration through downregulation of the PI3K/AKT pathway. Tumour Biol 35: 5189-5198, 2014.

44. Qiu FN, Huang Y, Chen DY, Li F, Wu YA, Wu WB and Huang XL: Eukaryotic elongation factor- $1 \alpha 2$ knockdown inhibits hepatocarcinogenesis by suppressing PI3K/Akt/NF- $\mathrm{B}$ signaling. World J Gastroenterol 22: 4226-4237, 2016.

45. Zhao J, Dong QZ, Zhong F, Cai LL, Qin ZY, Liu Y, Lin CZ, Qin LX and He FC: NMI promotes hepatocellular carcinoma progression via BDKRB2 and MAPK/ERK pathway. Oncotarget 8: 12174-12185, 2017.

46. Li J, Chen Y, Guo X, Zhou L, Jia Z, Tang Y, Lin L, Liu W and Ren C: Inhibition of miR-15b decreases cell migration and metastasis in colorectal cancer. Tumour Biol 37: 8765-8773, 2016.

47. Wang L, Chen YJ, Xu K, Xu H, Shen XZ and Tu RQ: Circulating microRNAs as a fingerprint for endometrial endometrioid adenocarcinoma. PLoS One 9: e110767, 2014.

48. Fan L, Qi H, Teng J, Su B, Chen H, Wang C and Xia Q: Identification of serum miRNAs by nano-quantum dots microarray as diagnostic biomarkers for early detection of non-small cell lung cancer. Tumour Biol 37: 7777-7784, 2016.

This work is licensed under a Creative Commons Attribution-NonCommercial-NoDerivatives 4.0 International (CC BY-NC-ND 4.0) License. 